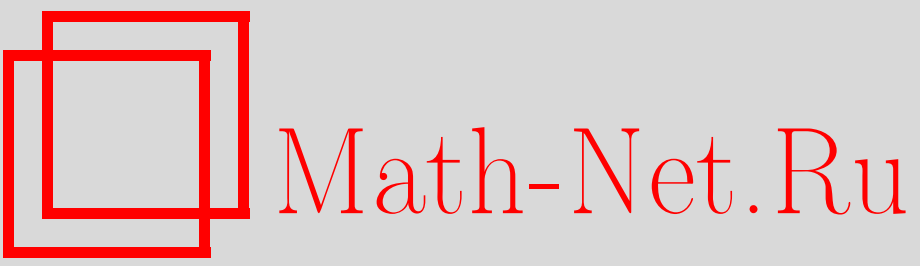

И. А. Ибрагимов, Н. В. Смородина, М. М. Фаддеев, Вероятностный подход к построению решений одномерных начально-краевых задач, Теория вероятн. и ее примен., 2013, том 58, выпуск 2, 255-281

DOI: https://doi.org/10.4213/tvp4506

Использование Общероссийского математического портала Math-Net.Ru подразумевает, что вы прочитали и согласны с пользовательским соглашением

http: //www . mathnet.ru/rus/agreement

Параметры загрузки:

IP : 3.89 .197 .203

26 апреля 2023 г., 15:04:22 


\title{
ВЕРОЯТНОСТНЫЙ ПОДХОД К ПОСТРОЕНИЮ РЕШЕНИЙ ОДНОМЕРНЫХ НАЧАЛЬНО-КРАЕВЫХ ЗАДАЧ ${ }^{1)}$
}

\begin{abstract}
В работе строится аналог вероятностного представления решения начально-краевой задачи для уравнения $\partial u / \partial t+\left(\sigma^{2} / 2\right) \partial^{2} u / \partial x^{2}+$ $f(x) u=0$, где $\sigma$ - комплексное число.

Ключевые слова и фразы: случайные процессы, эволюционное уравнение, предельная теорема, формула Фейнмана-Каца, интеграл Фейнмана, мера Фейнмана.
\end{abstract}

1. Введение. В настоящей работе строится аналог вероятностного представления решений ряда эволюционных уравнений. Пусть $[a, b]$ - интервал вещественной прямой, $\sigma$ - вещественное число. Для каждого $x \in[a, b]$ определим процесс $\xi_{x}(t)$, полагая

$$
\xi_{x}(t)=x+\sigma w(t),
$$

где $w(t), t \geqslant 0,-$ стандартный винеровский процесс, $w(0)=0$. Через $\tau$ обозначим момент первого достижения процессом $\xi_{x}(t)$ границы интервала $[a, b]$ и определим остановленный в этот момент процесс $\widetilde{\xi}_{x}(t)$, полагая

$$
\widetilde{\xi}_{x}(t)=\xi_{x}(t \wedge \tau) .
$$

Известно (см. [1], [3], [4]), что для всякой непрерывной на $[a, b]$ функции $\varphi$ функция

$$
u(t, x)=\mathbf{E} \varphi\left(\widetilde{\xi}_{x}(t)\right)
$$

*ПОМИ РАН, Санкт-Петербургский государственный университет, СанктПетербург, Россия; e-mail: ibr32@pdmi.ras.ru

** Санкт-Петербургский государственный университет, Санкт-Петербург, Россия; e-mail: smorodin@ns2691.spb.ru

*** Санкт-Петербургский государственный университет, Санкт-Петербург, Россия; e-mail: mmf@ns2691.spb.ru, mmfaddeev@gmail.com

1) Работа первого автора выполнена при поддержке программы «Ведущие научные школы» (грант НШ-1216.2012.1) и программы фундаментальных исследований РАН «Современные проблемы теоретической математики». Работа второго автора выполнена при поддержке РФФИ (грант № 12-01-00487a) и программы «Ведущие научные школы» (гранты НШ-1216.2012.1, НШ-357.2012.1). Работа третьего автора выполнена при поддержке РФФИ (гранты № 09-01-00515a, 11-01-90402укр-ф-а) и программы «Ведущие научные школы» (грант НШ-357.2012.1). 
является решением начально-краевой задачи для уравнения теплопроводности

$$
\frac{\partial u}{\partial t}=\frac{\sigma^{2}}{2} \frac{\partial^{2} u}{\partial x^{2}}, \quad u(0, x)=\varphi(x), \quad u(t, a)=\varphi(a), \quad u(t, b)=\varphi(b) .
$$

Таким образом, решение задачи (2) имеет вероятностное представление в виде математического ожидания функционала от остановленного (в момент первого достижения границы) процесса, а тем самым, семейство одномерных распределений процесса $\widetilde{\xi}_{x}(t), t \geqslant 0$, есть фундаментальное решение (2). В качестве вероятностного пространства в (1) можно взять пространство $C_{0}[0, T]$ непрерывных функций $h(t)$ таких, что $h(0)=0$, с винеровской мерой $P_{\sigma}$. Мера $P_{\sigma}$ однозначно продолжается с алгебры цилиндрических множеств на $\sigma$-алгебру борелевских множеств $C_{0}[0, T]$. При этом для каждого набора моментов времени $0<t_{1}<\cdots<t_{n} \leqslant T$ и каждого борелевского множества $A \subset \mathbf{R}^{n}$ мера $P_{\sigma}\left(U_{t_{1}, \ldots, t_{n}, A}\right)$ цилиндрического множества $U_{t_{1}, \ldots, t_{n}, A}$ вида

$$
U_{t_{1}, \ldots, t_{n}, A}=\left\{h \in C_{0}[0, T]:\left(h\left(t_{1}\right), \ldots, h\left(t_{n}\right)\right) \in A\right\}
$$

равна

$$
\frac{1}{\sigma^{n}(2 \pi)^{n / 2} \prod_{j=1}^{n}\left(t_{j}-t_{j-1}\right)^{1 / 2}} \int_{A} \exp \left(-\frac{1}{2 \sigma^{2}} \sum_{j=1}^{n} \frac{\left(x_{j}-x_{j-1}\right)^{2}}{t_{j}-t_{j-1}}\right) d x_{1} \cdots d x_{n}
$$

В случае, если на границе интервала вместо условия Дирихле ставится условие Неймана

$$
\frac{\partial u}{\partial x}(t, a)=\varphi^{\prime}(a), \quad \frac{\partial u}{\partial x}(t, b)=\varphi^{\prime}(b)
$$

соответствующее решение также допускает вероятностное представление (1) (на том же самом вероятностном пространстве), только вместо остановленного процесса следует использовать отраженный от границы процесс (см. [2], [3]).

В настоящей работе строится аналог вероятностного представления (1) для решения начально-краевой задачи (2) (с условием Дирихле или с условием Неймана) для случая комплексного $\sigma$, удовлетворяющего условию $\operatorname{Re} \sigma^{2} \geqslant 0$. Случай вещественного $\sigma$ соответствует уравнению теплопроводности, а случай $\operatorname{Re} \sigma^{2}=0$ - уравнению Шрёдингера. Кроме уравнения (1) рассматривается также начально-краевая задача для уравнения

$$
\frac{\partial u}{\partial t}=\frac{\sigma^{2}}{2} \frac{\partial^{2} u}{\partial x^{2}}+f(x) u
$$


возмущенного ограниченным потенциалом $f(x)$. В случае вещественного $\sigma$ решение задачи (4) может быть представлено формулой Фейнмана-Каца (для остановленного процесса), а именно,

$$
u(t, x)=\mathbf{E}\left\{\varphi\left(\widetilde{\xi}_{x}(t)\right) \exp \left(\int_{0}^{\tau} f\left(\widetilde{\xi}_{x}(v)\right) d v\right)\right\} .
$$

Известно [5], что для невещественных $\sigma$ не существует аналога винеровской меры $P_{\sigma}$ в том смысле, что мера, определенная на цилиндрических множествах формулой (3), не допускает продолжения на борелевскую $\sigma$-алгебру и, соответственно, невозможно представить формулу Фейнмана-Каца как интеграл относительно $\sigma$-аддитивной меры в пространстве траекторий. В случае, когда $\operatorname{Re} \sigma^{2}=0$ (что соответствует уравнению Шрёдингера), можно использовать интеграл по так называемой мере Фейнмана, которая является конечно-аддитивной мерой на пространстве траекторий. Соответствующий интеграл (интеграл Фейнмана) определяется через предел по измельчающейся последовательности разбиений интервала $[0, T]$. Следует отметить, что данный подход не является в обычном смысле вероятностным, так как в нем отсутствует само понятие вероятностного пространства (см. [6], [7]).

Имеется большое количество публикаций, посвященных различным подходам к построению интеграла Фейнмана. Один из рассматриваемых подходов, появившийся в работах [8]-[10], основан на аналитическом продолжении (по $\sigma$ ) в формуле Фейнмана-Каца. К сожалению, этот подход требует аналитичности потенциала, что является весьма ограничительным условием, а кроме того, этот подход не может быть взят за основу вероятностной конструкции приближения интеграла Фейнмана, так как требует введения понятия аналитической функции, аргумент которой сам является непрерывной функцией.

Альтернативный подход к математическому построению интеграла Фейнмана (см. [9], [11]-[13]) основан на построении комплекснозначной меры, дающей представление решения в виде функционального интеграла, но не самого решения уравнения Шрёдингера, а его преобразования Фурье. Данное представление по форме сильно отличается от исходной формулы Фейнмана-Каца для решения уравнения теплопроводности.

Мы в настоящей работе не ограничиваемся случаем $\operatorname{Re} \sigma^{2}=0$, a сразу рассматриваем все значения $\sigma$, удовлетворяющие условию $\arg \sigma \in$ $[-\pi / 4, \pi / 4]$. Это, в частности, дает возможность проследить эффекты, возникающие при $\arg \sigma \rightarrow \pm \pi / 4 \mp 0$.

Для построения аналогов вероятностных представлений (1) и (5) в настоящей работе используется другой подход, предложенный в [14], [15] и основанный на идеологии теории обобщенных функций. Мы будем рассматривать случайные (вообще говоря, комплекснозначные) процессы, 
но на распределения случайных величин мы будем смотреть не как на образ вероятностной меры, а как на образ обобщенной функции. Поясним подробнее, что имеется в виду. Пусть сначала $\xi$ - вещественнозначная случайная величина, заданная на вероятностном пространстве $(\Omega, \mathscr{F}, \mathbf{P})$, а $\mathscr{P}_{\xi}=\mathbf{P} \xi^{-1}-$ распределение этой случайной величины, т.е. образ меры $\mathbf{P}$ под действием отображения $\omega \mapsto \xi(\omega)$. Можно также смотреть на $\mathbf{P}$ как на обобщенную функцию, действующую на основную функцию $f$ как $\int_{\Omega} f d \mathbf{P}$. В этом случае определим распределение случайной величины $\xi$ как образ $\xi \mathbf{P}$ обобщенной функции $\mathbf{P}$ под действием $\xi$. По определению (см. [16]), $\xi \mathbf{P}$ есть обобщенная функция, которая на основную функцию $\varphi$ действует следующим образом:

$$
(\xi \mathbf{P}, \varphi)=(\mathbf{P}, \varphi \circ \xi)=\int_{\Omega} \varphi(\xi) d \mathbf{P}=\mathbf{E} \varphi(\xi) .
$$

Таким образом, для вещественной случайной величины $\xi$ понятия ее распределения как образа $\mathscr{P}_{\xi}=\mathbf{P} \xi^{-1}$ меры и как образа $\xi \mathbf{P}$ обобщенной функции совпадают. Именно, для каждой основной функции $\varphi$ мы имеем

$$
(\xi \mathbf{P}, \varphi)=\mathbf{E} \varphi(\xi)=\int_{\mathbf{R}} \varphi d \mathscr{P}_{\xi}
$$

Рассмотрим теперь другую ситуацию. Пусть для каждой функции $\varphi$ из заранее выбранного класса основных функций, заданных на некотором множестве $U$ (в нашем случае $U$ будет интервалом на вещественной прямой), определено ее продолжение $\widetilde{\varphi}$ на более широкую область $\widetilde{U}$. В данной работе мы будем рассматривать четные, нечетные, периодические и аналитические продолжения (разумеется, можно рассматривать и другие). Пусть у нас имеется случайная величина $\xi$, принимающая значения в $\widetilde{U}$. Посмотрим на распределение случайной величины $\xi$ как на образ обобщенной функции, т.е. как на обобщенную функцию. По определению, обобщенная функция $\xi \mathbf{P}$ действует на основную функцию $\varphi$ следующим образом:

$$
(\xi \mathbf{P}, \varphi)=\mathbf{E} \widetilde{\varphi}(\xi) .
$$

Если продолжения не было, т.е. $U=\widetilde{U}$ и $\varphi=\widetilde{\varphi}$, то справедлива формула (6), т.е. $\boldsymbol{\xi} \mathbf{P}$ есть обычное вероятностное распределение. Если же продолжение было нетривиально, то $\xi \mathbf{P}$, вообще говоря, уже не является вероятностной мерой. Важно отметить, что введенный нами объект $\xi \mathbf{P}$ - распределение случайной величины и в смысле обобщенных функций определяется не только случайной величиной $\xi$ и вероятностной мерой $\mathbf{P}$, но также и выбранным способом продолжения $\varphi \mapsto \widetilde{\varphi}$.

В качестве иллюстрации рассмотрим, как выглядит распределение случайной величины в смысле обобщенных функций для конкретного а именно, аналитического - продолжения. 
Сначала рассмотрим только наиболее интересный для нас случай, когда комплекснозначная случайная величина $\xi$ имеет вид $\xi=\sigma \eta$, где $\eta$ - вещественная случайная величина, а $\sigma$ - комплексная константа, причем $\operatorname{Im} \sigma \neq 0$. В этом случае распределение $\mathscr{P}_{\xi}=\mathbf{P} \xi^{-1}$ случайной величины $\xi$, рассматриваемое как образ меры $\mathbf{P}$, есть вероятностная мера на комплексной плоскости, носитель которой лежит на прямой $\sigma t$, $t \in \mathbf{R}$.

Предположим дополнительно, что $\mathbf{E} e^{\lambda|\eta|}<\infty$ для любого $\lambda \in \mathbf{R}$, и найдем распределение $\xi$ в смысле обобщенных функций (т.е. как обобщенную функцию, определенную формулой (7)), предполагая, что в (7) $U=\mathbf{R}, \widetilde{U}=\mathbf{C}$, а в качестве продолжения $\widetilde{\varphi}$ функции $\varphi$ мы берем ее аналитическое продолжение.

В качестве пространства основных функций мы возьмем множество (далее будем обозначать его $\mathscr{H}_{\text {fin }}$ ) комплекснозначных функций $\varphi$ вещественного аргумента, которые являются преобразованиями Фурье конечного заряда с финитным носителем. По определению, обобщенная функция $\xi \mathbf{P}$ действует на основную функцию $\varphi \in \mathscr{H}_{\text {fin }}$ в соответствии с формулой $(7)$, при этом в $(7)$ функция $\widetilde{\varphi}(z)$ комплексной переменной $z$ (аналитическое продолжение $\varphi$ ) задается формулой

$$
\widetilde{\varphi}(z)=\frac{1}{2 \pi} \int_{\mathbf{R}} e^{-i p z} \widehat{\varphi}(p) d p
$$

Таким образом, в данном случае мы имеем

$$
\begin{aligned}
(\xi \mathbf{P}, \varphi) & =\mathbf{E} \widetilde{\varphi}(\xi)=\frac{1}{2 \pi} \mathbf{E} \int_{\mathbf{R}} e^{-i p \xi} \widehat{\varphi}(p) d p \\
& =\frac{1}{2 \pi} \int_{\mathbf{R}} \widehat{\varphi}(p) \mathbf{E} e^{-i p \sigma \eta} d p=\frac{1}{2 \pi} \int_{\mathbf{R}} \widehat{\varphi}(p) \int_{\mathbf{R}} e^{-i p \sigma y} d \mathscr{P}_{\eta}(y) d p .
\end{aligned}
$$

Последнее выражение показывает, что преобразование Фурье $\widehat{\xi \mathbf{P}}$ обобщенной функции $\xi \mathbf{P}$ имеет вид $f_{\eta}(\sigma p)$, где $f_{\eta}(p)=\mathbf{E} e^{i p \eta}-$ характеристическая функция случайной величины $\eta$. В частности, если случайная величина $\eta$ имеет стандартное нормальное распределение, a $\operatorname{Re} \sigma^{2} \geqslant 0$, то распределение случайной величины $\xi=\sigma \eta$ в смысле обобщенных функций есть регулярный функционал с плотностью $e^{-x^{2} /\left(2 \sigma^{2}\right)} /(\sigma \sqrt{2 \pi})$.

Аналогичным образом, в случае, когда $\xi$ - произвольная комплекснозначная случайная величина (не обязательно имеющая вид вещественной случайной величины, умноженной на комплексную константу), формулы (7), (8) задают обобщенную функцию $\xi \mathbf{P}$, имеющую преобразование Фурье $\widehat{\xi \mathbf{P}}(p)=\mathbf{E} e^{i p \xi}$.

Важно отметить, что распределение комплекснозначной случайной величины, задаваемое формулами (7), (8), по определению является 
только обобщенной функцией, исходно заданной на очень узком классе основных функций. Если же нам дополнительно известно, что соответствующее преобразование Фурье достаточно быстро убывает, то мы можем продолжить $\xi \mathbf{P}$ на более широкий класс функций.

Далее, данный подход легко обобщается на векторный случай, именно на случай, когда $\xi$ есть случайный элемент $\mathbf{C}^{d}$ : для этого в $(7),(8)$ в качестве основных функций $\varphi$ надо использовать аналитические функции нескольких переменных [16]. Представляется естественным, используя формулу (7), попытаться для комплексных $\sigma$ определить распределение процесса $\sigma w(t), t \in[0, T]$, как обобщенную функцию, заданную на классе основных функций, зависящих от всей траектории процесса, но при этом возникают трудности, связанные с определением понятия аналитической функции $\varphi(z)$, аргумент $z$ которой, в свою очередь, является непрерывной комплекснозначной функцией. Одним из возможных способов преодоления этой трудности, по-видимому, является подход с использованием техники кратных стохастических интегралов, но мы в настоящей работе используем другой подход, основанный на идеях работы [15]. Именно, вместо винеровского процесса используется некоторый обобщенный случайный процесс. В отличие от обычного вероятностного процесса обобщенный случайный процесс задается не на вероятностном пространстве, а на пространстве, на котором вместо вероятностной меры задана некоторая обобщенная функция. Соответственно, конечномерные распределения обобщенного процесса суть уже не образы вероятностной меры, а образы обобщенной функции (т.е. тоже обобщенные функции). В качестве заменителя винеровского процесса мы выбираем обобщенный процесс, конечномерные распределения которого совпадают с конечномерными распределениями винеровского процесса. Преимущество использования обобщенного процесса вместо винеровского состоит в том, что все его траектории устроены очень просто. Именно, они всегда состоят из конечного числа скачков, соответственно, аналитическая функция от траекторий этого процесса есть просто аналитическая функция конечного числа переменных. В терминах нашего обобщенного процесса мы строим представления решений вида (1), где вместо операции взятия математического ожидания используется вычисление обобщенной функции. Дополнительно мы строим аппроксимацию обобщенного процесса последовательностью $\xi_{n}(t), t \in[0, T]$, сложных пуассоновских процессов. Последовательность $\xi_{n}$, с одной стороны, слабо сходится (в пространстве Скорохода с топологией, порожденной равномерной нормой) к винеровскому процессу, а с другой стороны, в смысле обобщенных функций (т.е. на каждой основной функции) последовательность мер, порожденных $\xi_{n}$, сходится к нашему обобщенному процессу, который мы выбрали в качестве заменителя винеровского процесса. 
2. Основные обозначения. Пусть $\delta$ обозначает обобщенную функцию, которая на основную функцию $\varphi$ действует как $(\delta, \varphi)=\varphi(0)$. Через $\delta^{(n)}$ будем обозначать производные этой обобщенной функции, именно, $\left(\delta^{(n)}, \varphi\right)=(-1)^{n} \varphi^{(n)}(0)$.

Далее, для $t \in \mathbf{R}$ через $\delta_{t}$ будем обозначать $\delta$-меру в точке $t$. На этот объект нам будет удобнее смотреть именно как на меру, а не как на обобщенную функцию.

Через $\mathscr{R}[-\pi, \pi]$ будем обозначать пространство ограниченных (комплекснозначных) $2 \pi$-периодических функций $f$ таких, что

$$
f(x)=\sum_{m=-\infty}^{\infty} B_{m} e^{i m x}, \quad \sum_{m=-\infty}^{\infty}\left|B_{m}\right|<\infty,
$$

где $B_{m}-$ коэффициенты ряда Фурье функции $f$. Норма $\|\cdot\|_{\mathscr{R}}$ в этом пространстве определяется формулой $\|f\|_{\mathscr{R}}=\sum_{m=-\infty}^{\infty}\left|B_{m}\right|$. Справедливо неравенство $\|f\|_{\infty} \leqslant\|f\|_{\mathscr{R}}$. Если функция $f$ исходно задана на интервале $[-\pi, \pi]$, то той же самой буквой $f$ мы будем обозначать и ее $2 \pi$ периодическое продолжение.

Через $\mathscr{R}^{(l)}[-\pi, \pi]$ обозначим подпространство $\mathscr{R}[-\pi, \pi]$ функций, для которых $\sum_{m=-\infty}^{\infty}|m|^{l}\left|B_{m}\right|<\infty$. Каждая функция из $\mathscr{R}^{(l)}[-\pi, \pi]$ является, вместе со своими производными до порядка $l$ включительно, непрерывной $2 \pi$-периодической функцией.

3. Обобщенная функция как предел вероятностных мер. В этом разделе мы изложим конструкцию, предложенную в [15]. Зафиксируем $T>0$ и через $\Omega_{0}$ обозначим пространство всех дискретных зарядов на $[0, T]$ с конечным числом атомов. Каждый элемент $\omega$ этого пространства представляется в виде

$$
\omega=\sum_{j=1}^{k} x_{j} \delta_{t_{j}},
$$

где $k \in \mathbf{N} \cup\{0\}-$ число атомов заряда $\omega, \delta_{t_{j}}-\delta$-мера, сосредоточенная в точке $t_{j} \in[0, T]$, а $x_{j}$ есть величина заряда, «сидящего» в точке $t_{j}$. Заметим, что представление (10) будет однозначным, если мы условимся, что в данном представлении $\left|x_{j}\right|>0$ для всех $j$ и все точки $t_{j}$ различны.

Для $\omega \in \Omega_{0}$ и $0 \leqslant a<b \leqslant T$ через $\omega[a, b]$ будем обозначать заряд интервала $[a, b]$, т.е. $\omega[a, b]=\sum_{j} x_{j}$, где суммирование проводится по тем индексам $j$, для которых $t_{j} \in[a, b]$.

Пространство $\Omega_{0}$ (как подпространство пространства зарядов) является, естественным образом, линейным пространством - умножение на число понимается как умножение заряда на число, а сложение тоже понимается как сложение зарядов. На $\Omega_{0}$ мы рассмотрим топологию, в которой сходящимися будут слабо сходящиеся последовательности зарядов. Через $\mathscr{B}\left(\Omega_{0}\right)$ обозначим борелевскую $\sigma$-алгебру на $\Omega_{0}$. 
На пространстве $\Omega_{0}$ определим два объекта - последовательность вероятностных мер $\left\{\mathbf{P}_{n}\right\}_{n=1}^{\infty}$ и обобщенную функцию $\mathbf{L}$. При этом обобщенная функция $\mathbf{L}$ окажется пределом последовательности $\mathbf{P}_{n}$ в смысле сходимости обобщенных функций (заметим здесь, что всякую меру мы можем рассматривать двояко - и как меру, и как обобщенную функцию). Определим сначала последовательность вероятностных мер $\mathbf{P}_{n}$. Каждая мера $\mathbf{P}_{n}$ порождается некоторым сложным пуассоновским процессом. Именно, пусть $\nu_{n}$ - пуассоновская случайная мера на $[0, T]$ с интенсивностью $n d t$, т.е. $\mathbf{E} \nu_{n}(d t)=n d t$, а $\left\{\xi_{j}\right\}_{j=1}^{\infty}$ - ортогауссовская последовательность случайных величин, не зависящая от пуассоновской случайной меры.

Для каждого натурального $n$ построим случайный заряд $\zeta_{n}$ (случайный элемент $\Omega_{0}$ ) следующим образом. Пусть

$$
\nu_{n}=\sum_{j=1}^{k} \delta_{t_{j}}, \quad t_{1}<t_{2}<\cdots<t_{k},
$$

- реализация пуассоновской случайной меры $\nu_{n}$ (здесь $k=\nu_{n}[0, T]$ ). В каждую точку $t_{j}, j=1, \ldots, k$, поместим заряд $\xi_{j} / \sqrt{n}$. То, что получилось, будет случайным зарядом, который обозначим через $\zeta_{n}$, таким образом,

$$
\zeta_{n}=\sum_{j=1}^{k} \frac{\xi_{j}}{\sqrt{n}} \delta_{t_{j}}, \quad \zeta_{n} \in \Omega_{0} .
$$

Пусть $\mathbf{P}_{n}$ обозначает распределение случайного заряда $\zeta_{n}$ в $\Omega_{0}$, а $\mathbf{E}_{n}$ - математическое ожидание по мере $\mathbf{P}_{n}$.

Приведем удобную формулу для вычисления интегралов по мере $\mathbf{P}_{n}$. Пусть $f: \Omega_{0} \rightarrow \mathbf{C}$ - измеримая (по Борелю) комплекснозначная функция. Для каждой такой функции $f$ через $f_{k}, k \in \mathbf{N} \cup\{0\}$, обозначим симметричную функцию $k$ двумерных переменных, определяемую формулой

$$
f_{k}\left(\left(t_{1}, x_{1}\right),\left(t_{2}, x_{2}\right), \ldots,\left(t_{k}, x_{k}\right)\right)=f\left(\sum_{j=0}^{k} x_{j} \delta_{t_{j}}\right) .
$$

При различных $k$ функции $f_{k}$ связаны соотношениями

$$
f_{k+1}\left(\left(t_{1}, x_{1}\right), \ldots,\left(t_{k}, x_{k}\right),\left(t_{k+1}, 0\right)\right)=f_{k}\left(\left(t_{1}, x_{1}\right), \ldots,\left(t_{k}, x_{k}\right)\right) .
$$

Таким образом, задание функции $f: \Omega_{0} \rightarrow \mathbf{C}$ эквивалентно заданию последовательности $\left\{f_{k}\right\}_{k=0}^{\infty}$ симметричных функций, связанных соотношениями (12). При этом $f_{0}=$ const, а при $k>0$ функция $f_{k}$ является симметричной функцией $k$ двумерных переменных.

Далее $g_{n}$ будет обозначать обобщенную функцию, которая на основную функцию $\psi: \mathbf{R} \rightarrow \mathbf{C}$ (классы основных функций мы будем вводить 
по мере необходимости) действует следующим образом:

$$
\left(g_{n}, \psi\right)=\frac{n}{\sqrt{2 \pi}} \int_{\mathbf{R}}\left(\psi\left(\frac{y}{\sqrt{n}}\right)-\psi(0)\right) e^{-y^{2} / 2} d y .
$$

Заметим, что если функция $\psi$ имеет две непрерывные ограниченные производные, то

$$
\lim _{n \rightarrow \infty}\left(g_{n}, \psi\right)=\frac{\psi^{(2)}(0)}{2}=\left(\frac{\delta^{(2)}}{2}, \psi\right)
$$

т.е. обобщенная функция $\delta^{(2)} / 2$ есть предел обобщенных функций $g_{n}$.

Для каждого $k \in \mathbf{N}$ через $m^{k}$ будем обозначать меру Лебега на $[0, T]^{k}$, а через $g_{n}^{\otimes k}-$ тензорное произведение (см. [16]) обобщенных функций $g_{n} \otimes g_{n} \otimes \cdots \otimes g_{n}$.

Теорема 1. Для всякой измеримой ограниченной функции $f: \Omega_{0} \rightarrow$ С имеет место соотношение

$$
\int_{\Omega_{0}} f d \mathbf{P}_{n}=\mathbf{E}_{n} f=\sum_{k=0}^{\infty} \frac{1}{k !} \int_{[0, T]^{k}}\left(g_{n}^{\otimes k}, f_{k}\right) d m^{k}
$$

Здесь при каждом $k>0$ интегрирование по мере Лебега ведется по временньм переменным $t_{j} \in[0, T]$, а обобщенная функиия $g_{n}^{\otimes k}$ действует по пространственным переменным $x_{j} \in \mathbf{R}$. Слагаемое с $k=0$ равно $f_{0}$.

Доказательство можно найти в [15].

Далее мы будем рассматривать не только интервал $[0, T]$, но и различные его подынтервалы. Введем соответствующие определения. Пусть $[t, s) \subset[0, T]$. Через $\Omega_{0}^{t, s}$ мы обозначим множество дискретных зарядов с конечным спектром на интервале $[t, s)$. Ясно, что для любого $u \in(t, s)$ множество $\Omega_{0}^{t, s}$ естественно изоморфно декартову произведению $\Omega_{0}^{t, u} \times \Omega_{0}^{u, s}$. Обозначим через $\mathbf{P}_{n}^{t, s}$ сужение меры $\mathbf{P}_{n}$ на $\Omega_{0}^{t, s}$. Ясно, что

$$
\mathbf{P}_{n}^{t, s}=\mathbf{P}_{n}^{t, u} \times \mathbf{P}_{n}^{u, s}
$$

где символ $\times$ обозначает прямое произведение соответствующих мер.

Итак, последовательность вероятностных мер у нас построена. Определим теперь обобщенную функцию L. Для каждой функции $f: \Omega_{0} \rightarrow \mathbf{C}$, принадлежащей некоторому множеству основных функций $\mathscr{G}$ (множество основных функций мы опишем чуть позднее), положим

$$
\mathbf{L} f=(\mathbf{L}, f)=\sum_{k=0}^{\infty} \frac{1}{k !} \int_{[0, T]^{k}}\left(\left(\frac{\delta^{(2)}}{2}\right)^{\otimes k}, f_{k}\right) d m^{k} .
$$


Здесь, как и ранее, $m^{k}$ обозначает мер Лебега на $[0, T]^{k}$, причем интегрирование ведется по временным переменным $t_{j} \in[0, T]$, а обобщенная функция $\left(\delta^{(2)} / 2\right)^{\otimes k}$ действует по пространственным переменным $x_{j} \in \mathbf{R}$.

Отметим еще, что если $f \equiv f_{0}=$ const, то

$$
\mathbf{L} f=f_{0},
$$

так как в сумме (16) отличным от нуля будет только слагаемое с $k=0$.

Опишем теперь пространство основных функций $\mathscr{G}$. Каждая основная функция $f \in \mathscr{G}$ есть измеримая функция из $\Omega_{0}$ в $\mathbf{C}$; через $f_{k}$, $k \in \mathbf{N} \cup\{0\}$, как и ранее, будем обозначать функции, определяемые равенством (11).

Первым шагом для каждого $k \geqslant 1$ и $A>0$ определим на множестве функций $h:([0, T] \times \mathbf{R})^{k} \rightarrow \mathbf{C}$ нормы $\|\cdot\|_{k, A}$, полагая

$$
\|h\|_{k, A}=\sum_{I \subset\{1, \ldots, k\}} \int_{[0, T]^{k}} d m^{k} \max _{\substack{p \\ 0 \leqslant p_{j} \leqslant 3}} \sup _{\substack{\left|x_{i}\right| \leqslant 1, j \in I}} \sup _{\substack{\left|x_{j}\right|>1, j \notin I}}\left(\left|\mathscr{D}_{I, p} h\right| \exp \left(-A \sum_{j \notin I}\left|x_{j}\right|\right)\right),
$$

где для $I \subset\{1, \ldots, k\}$ и $p=\left(p_{1}, \ldots, p_{k}\right) \in \mathbf{N}^{k}$ через $\mathscr{D}_{I, p}$ обозначен дифференциальный оператор вида

$$
\mathscr{D}_{I, p}=\prod_{j \in I} \frac{\partial^{p_{j}}}{\partial x^{p_{j}}},
$$

причем мы всюду в (18) предполагаем, что операторы $\mathscr{D}_{I, p}$ действуют только по переменным $x_{i}$. Смысл введенной нормы простой - по переменным $x_{j} \in[-1,1]$ должны быть ограничены сама функция и три ее первых производных, а на бесконечности функция должна возрастать не быстрее, чем $e^{A\left|x_{j}\right|}$.

Определим теперь пространство $\mathscr{G}$ основных функций как множество измеримых функций $f: \Omega_{0} \rightarrow \mathbf{C}$ таких, что для каждого $k>0$ функция $f_{k}=f_{k}\left(\left(t_{1}, x_{1}\right), \ldots,\left(t_{k}, x_{k}\right)\right)$ бесконечно дифференцируема по переменным $x_{j}$, а для всех $M>0$ и некоторого $A>0$

$$
\sum_{k=0}^{\infty} \frac{M^{k}\left\|f_{k}\right\|_{k, A}}{k !}<\infty
$$

Отметим, что условие (20) гарантирует сходимость ряда (16).

Теорема 2. Для любой $f \in \mathscr{G}$

$$
\lim _{n \rightarrow \infty} \int_{\Omega_{0}} f d \mathbf{P}_{n}=\mathbf{L} f
$$

или, другими словами, в смысле обобщенных функций

$$
\lim _{n \rightarrow \infty} \mathbf{P}_{n}=\mathbf{L} \text {. }
$$


Фактически утверждение теоремы следует из формулы (14). Условие (20) дает нам возможность совершить предельный переход под знаком суммы. Подробное доказательство можно найти в [15].

Далее, как мы уже отмечали, для любых $u \in(t, s)$ множество $\Omega_{0}^{t, s}$ естественно изоморфно декартову произведению $\Omega_{0}^{t, u} \times \Omega_{0}^{u, s}$. Для $t<s$ через $\mathbf{L}^{t, s}$ будем обозначать сужение обобщенной функции $\mathbf{L}$ на $\Omega_{0}^{t, s}$, понимая под этим обобщенную функцию, определенную на подмножестве $\mathscr{G}^{t, s} \subset \mathscr{G}$ основных функций, каждая из которых зависит только от той части заряда, которая попала в интервал $[t, s)$, т.е. любая функция $f \in \mathscr{G}^{t, s}$ удовлетворяет условию $f(\omega)=f\left(\left.\omega\right|_{[t, s)}\right)$ для всех $\omega \in \Omega_{0}$. На основную функцию $f \in \mathscr{G}^{t, s}$ обобщенная функция $\mathbf{L}^{t, s}$ действует следующим образом:

$$
\mathbf{L}^{t, s} f=\sum_{k=0}^{\infty} \frac{1}{k !} \int_{[t, s]^{k}}\left(\left(\frac{\delta^{(2)}}{2}\right)^{\otimes k}, f_{k}\right) d m^{k}
$$

Нетрудно показать, что для любых $t<u<s$ справедливо соотношение

$$
\mathbf{L}^{t, s}=\mathbf{L}^{t, u} \otimes \mathbf{L}^{u, s}
$$

Итак, мы построили предельный объект $\left(\Omega_{0}, \mathscr{G}, \mathbf{L}\right)$, который не является вероятностным пространством, и последовательность обычных вероятностных мер $\mathbf{P}_{n}$ на $\left(\Omega_{0}, \mathscr{B}\left(\Omega_{0}\right)\right)$. На каждое $\mathbf{P}_{n}$ мы можем смотреть и как на меру, и как на обобщенную функцию, заданную на множестве $\mathscr{G}$ основных функций. При этом в смысле обобщенных функций (т.е. на каждой основной функции) последовательность $\mathbf{P}_{n}$ сходится к $\mathbf{L}$.

4. Процессы с независимыми приращениями и порожденные ими полугруппы операторов. Обозначим через $D_{0}([0, T], \mathbf{C})$ множество непрерывных справа кусочно-постоянных комплекснозначных функций с конечным числом скачков. На этом множестве рассмотрим топологию, индуцированную (как на подмножестве пространства Скорохода) топологией Скорохода (см. [17]) и соответствующую борелевскую $\sigma$-алгебру.

Обобщенным случайным процессом (слово обобщенный далее будет опускаться) мы будем называть любое измеримое отображение из $\Omega_{0}$ в множество $D_{0}([0, T], \mathbf{C})$. Заметим, что процесс будет обобщенным, только если на $\Omega_{0}$ мы рассматриваем обобщенную функцию L. B допредельном случае мы имеем дело с обычным случайным процессом, заданным на вероятностном пространстве $\left(\Omega_{0}, \mathscr{B}\left(\Omega_{0}\right), \mathbf{P}_{n}\right)$.

Далее, пусть $\sigma \in \mathbf{C},|\sigma|=1$, Re $\sigma^{2} \geqslant 0$, - фиксированное комплексное число. Для каждого $x \in \mathbf{R}$ определим случайный процесс $\xi_{x}^{\sigma}(t)$, $t \in[0, T]$, полагая

$$
\xi_{x}^{\sigma}(t)=\xi_{x}^{\sigma}(t, \omega)=x+\sigma \omega[0, t]
$$


где $\omega[0, t]$ обозначает заряд $\omega$ интервала $[0, t]$.

Нетрудно понять, что для каждого фиксированного $n$ на вероятностном пространстве $\left(\Omega_{0}, \mathscr{B}\left(\Omega_{0}\right), \mathbf{P}_{n}\right)$ процесс $\xi_{x}^{\sigma}(t), t \in[0, T]$, является сложным пуассоновским процессом.

В работе [15] изучались одномерные распределения процесса $\xi_{x}^{\sigma}(t)$. При этом под одномерным распределением понимался либо $\xi_{x}^{\sigma}(t) \mathbf{L}$, т.е. образ обобщенной функции $\mathbf{L}$ под действием $\xi_{x}^{\sigma}(t)$, либо, если речь шла о допредельном случае, $\xi_{x}^{\sigma}(t) \mathbf{P}_{n}$ - соответствующий образ обобщенной функции $\mathbf{P}_{n}$. В качестве продолжения $\widetilde{\varphi}$ функции $\varphi$ в формуле (7) бралось ее аналитическое продолжение (8).

В качестве пространства основных функций для обобщенных функций $\xi_{x}^{\sigma}(t) \mathbf{L}$ и $\xi_{x}^{\sigma}(t) \mathbf{P}_{n}$ в [15] выбиралось множество $\mathscr{H}_{\text {fin }}$ функций $\varphi$, которые являются преобразованиями Фурье конечного заряда с финитным носителем. Именно, каждая функция $\varphi \in \mathscr{H}_{\text {fin }}$ имеет вид $\varphi(x)=$ $(2 \pi)^{-1} \int_{-A}^{A} e^{-i p x} \mu(d p)$, где $\mu-$ заряд на $[-A, A]$, удовлетворяющий условию $|\mu|([-A, A])<\infty$.

Любая $\varphi \in \mathscr{H}_{\text {fin }}$ является целой функцией экспоненциального типа, и, кроме того, для любых $x, t, \sigma$ функция $\omega \mapsto \widetilde{\varphi}\left(\xi_{x}^{\sigma}(t, \omega)\right)$ принадлежит $\mathscr{G}$.

Следуя [15], определим две полугруппы операторов $P_{n}^{t}$ и $P^{t}(t \geqslant 0)$, полагая для $\varphi \in \mathscr{H}_{\text {fin }}$

$$
\begin{aligned}
P^{t} \varphi(x) & =\mathbf{L} \widetilde{\varphi}\left(\xi_{x}^{\sigma}(t)\right)=\mathbf{L} \widetilde{\varphi}(x+\sigma \omega[0, t])=\mathbf{L}^{0, t} \widetilde{\varphi}(x+\sigma \omega[0, t]) \\
& =\sum_{k=0}^{\infty} \frac{t^{k}}{k !}\left(\left(\frac{\delta^{(2)}}{2}\right)^{\otimes k}, \widetilde{\varphi}\left(x+\sigma\left(x_{1}+\cdots+x_{k}\right)\right)\right), \\
P_{n}^{t} \varphi(x) & =\mathbf{E}_{n} \widetilde{\varphi}\left(\xi_{x}^{\sigma}(t)\right)=\sum_{k=0}^{\infty} \frac{t^{k}}{k !}\left(g_{n}^{\otimes k}, \widetilde{\varphi}\left(x+\sigma\left(x_{1}+\cdots+x_{k}\right)\right)\right) .
\end{aligned}
$$

Здесь $\mathbf{E}_{n}$, как и ранее, обозначает математическое ожидание по мере $\mathbf{P}_{n}$, а обобщенная функция $g_{n}$ определяется формулой (13).

Используя (24), нетрудно показать, что для каждой $\varphi \in \mathscr{H}_{\text {fin }}$ функция $u(t, x)=P^{t} \varphi(x)$ решает задачу Коши

$$
\frac{\partial u}{\partial t}=\frac{\sigma^{2}}{2} \frac{\partial^{2} u}{\partial x^{2}}, \quad u(0, x)=\varphi(x),
$$

а используя (25), также легко показать, что для каждой $\varphi \in \mathscr{H}_{\text {fin }}$ функция $u_{n}(t, x)=P_{n}^{t} \varphi(x)$ решает задачу Коши

$$
\frac{\partial u_{n}}{\partial t}=A_{n}^{\sigma} u_{n}, \quad u_{n}(0, x)=\varphi(x),
$$

где оператор $A_{n}^{\sigma}$ действует на функцию $\psi \in \mathscr{H}_{\text {fin }}$ следующим образом:

$$
A_{n}^{\sigma} \psi(x)=\frac{n}{\sqrt{2 \pi}} \int_{\mathbf{R}}\left(\psi\left(x+\frac{\sigma y}{\sqrt{n}}\right)-\psi(x)\right) e^{-y^{2} / 2} d y .
$$


Из теоремы 2 следует, что для любых фиксированных $t, x$ имеет место сходимость $u_{n}(t, x) \rightarrow u(t, x), n \rightarrow \infty$. В [15] рассматривались вопросы продолжения операторов $P_{n}^{t}, P^{t}$ с $\varphi \in \mathscr{H}_{\text {fin }}$ на более широкий класс функций $\varphi$, а также вопрос сходимости $u_{n} \rightarrow u$ в различных метриках.

$\mathrm{B}$ настоящей работе мы получим представление решения начальнокраевой задачи на ограниченном интервале. При этом мы не будем использовать, как в [3], остановленный в первый момент достижения границы (или отражающийся от границы) процесс. Наличие границы будет учтено другим способом - при помощи построения продолжения функции за рассматриваемый интервал. Эти продолжения будут различными для случаев условия Дирихле и условия Неймана.

4.1. Условие Дирихле. Для удобства будем проводить рассмотрения на интервале $[0, \pi]$. Общий случай сводится к этому линейной заменой аргумента. Итак, пусть непрерывная комплекснозначная функция $\varphi$ определена на интервале $[0, \pi]$. Разложим функцию $\varphi$ в сумму

$$
\varphi(x)=\varphi(0)+\frac{\varphi(\pi)-\varphi(0)}{\pi} x+\varphi_{0}(x) ;
$$

ясно, что при этом $\varphi_{0}(0)=\varphi_{0}(\pi)=0$. Для каждой функции $\varphi_{0}$ через $\varphi_{0}^{\text {odd }}$ обозначим ее нечетное продолжение с $[0, \pi]$ на $[-\pi, \pi]$.

Пусть $\mathscr{H}_{D}$ обозначает множество функций $\varphi:[0, \pi] \rightarrow \mathbf{C}$, у которых в разложении (28) функция $\varphi_{0}^{\text {odd }}$ имеет вид

$$
\varphi_{0}^{\text {odd }}(x)=\sum_{m=-\infty}^{\infty} B_{m} e^{i m x}
$$

причем $B_{0}=0$, для всех $m$ выполнено равенство $B_{-m}=-B_{m}$ и только конечное число коэффициентов $B_{m}$ отлично от нуля (т.е. $\varphi_{0}$ есть тригонометрический полином, являющийся конечной линейной комбинацией синусов).

Для функции $\varphi \in \mathscr{H}_{D}$ определим ее продолжение $\widetilde{\varphi}_{D}$ на комплексную плоскость, полагая для $z \in \mathbf{C}$

$$
\widetilde{\varphi}_{D}(z)=\varphi(0)+\frac{\varphi(\pi)-\varphi(0)}{\pi} z+\sum_{m=-\infty}^{\infty} B_{m} e^{i m z} .
$$

Отметим, что при этом продолжении с интервала $[0, \pi]$ на вещественную ось функция $\varphi_{0}$ продолжается сначала нечетным (на $\left.[-\pi, \pi]\right)$, а затем $2 \pi$-периодическим образом.

Определим две полугруппы операторов $P_{D, n}^{t}$ и $P_{D}^{t}(t \geqslant 0)$, полагая для $\varphi \in \mathscr{H}_{D}$

$$
\begin{aligned}
P_{D}^{t} \varphi(x) & =\mathbf{L} \widetilde{\varphi}_{D}\left(\xi_{x}^{\sigma}(t)\right)=\mathbf{L}^{0, t} \widetilde{\varphi}_{D}(x+\sigma \omega[0, t]), \\
P_{D, n}^{t} \varphi(x) & =\mathbf{E}_{n} \widetilde{\varphi}_{D}\left(\xi_{x}^{\sigma}(t)\right)=\mathbf{E}_{n} \widetilde{\varphi}_{D}(x+\sigma \omega[0, t]) .
\end{aligned}
$$

Посмотрим, как действует на функцию $\varphi \in \mathscr{H}_{D}$ оператор $P_{D}^{t}$. 
Лемма 1. Для любых $t \in[0, T], a \in \mathbf{C}$ справедливь следуюшие утверждения.

1. $\mathbf{L} \omega[0, t]=0$.

2. $\mathbf{L}(\omega[0, t])^{2}=t$.

3. $\mathbf{L} e^{i a \omega[0, t]}=e^{-t a^{2} / 2}$.

Д о к а з а т е л ь с т в о. Докажем только утверждение 3, так как первые два утверждения являются его следствиями. Прежде всего заметим, что $\mathbf{L} e^{i a \omega[0, t]}=\mathbf{L}^{0, t} e^{i a \omega[0, t]}$. Определим функцию $f: \Omega_{0}^{0, t} \rightarrow \mathbf{C}$, полагая $f(\omega)=e^{i a \omega[0, t]}$. Тогда, как легко видеть, $f_{0}=1$, а для всех $k>0$ выполнено равенство $f_{k}\left(\left(t_{1}, x_{1}\right), \ldots,\left(t_{k}, x_{k}\right)\right)=e^{i a\left(x_{1}+\cdots+x_{k}\right)}$. В силу $(21)$, (22) имеем

$$
\mathbf{L}^{0, t} e^{i a \omega[0, t]}=\sum_{k=0}^{\infty} \frac{t^{k}}{k !}\left(\left(\frac{\delta^{(2)}}{2}\right)^{\otimes k}, e^{i a\left(x_{1}+\cdots+x_{k}\right)}\right)=e^{-t a^{2} / 2} .
$$

Лемма 1 доказана.

Используя (17) и лемму 1, получим

$$
\begin{aligned}
P_{D}^{t} \varphi(x)= & \mathbf{L}^{0, t} \widetilde{\varphi}_{D}(x+\sigma \omega[0, t]) \\
= & \mathbf{L}^{0, t}\left(\varphi(0)+\frac{\varphi(\pi)-\varphi(0)}{\pi}(x+\sigma \omega[0, t])\right) \\
& +\mathbf{L}^{0, t}\left(\sum_{m=-\infty}^{\infty} B_{m} e^{i m(x+\sigma \omega[0, t])}\right) \\
= & \varphi(0)+\frac{\varphi(\pi)-\varphi(0)}{\pi} x+\sum_{m=-\infty}^{\infty} B_{m} e^{-t \sigma^{2} m^{2} / 2} e^{i m x},
\end{aligned}
$$

что означает, что оператор $P_{D}^{t}$ не меняет линейную часть функции $\varphi$, а его действие на $\varphi_{0}$ сводится к умножению коэффициента Фурье $B_{m}$ на величину $e^{-t \sigma^{2} m^{2} / 2}$ при всех $m \in \mathbf{Z}$.

Отметим, что исходно оператор $P_{D}^{t}$ задан только на функциях из множества $\mathscr{H}_{D}$. Однако из условия $\operatorname{Re} \sigma^{2} \geqslant 0$ вытекает, что, пользуясь формулой (31), мы можем продолжить его на множество функций $\varphi$ таких, что $\varphi_{0}^{\text {odd }} \in \mathscr{R}[-\pi, \pi]$, т.е. для тех $\varphi_{0}^{\text {odd }}$, для которых сходится ряд $\sum_{m=-\infty}^{\infty}\left|B_{m}\right|$. Если $\operatorname{Re} \sigma^{2}>0$, то для каждой такой функции $\varphi$ функция $u(t, x)=P_{D}^{t} \varphi(x)$ (здесь речь идет о продолженном операторе) является решением начально-краевой задачи

$$
\frac{\partial u}{\partial t}=\frac{\sigma^{2}}{2} \frac{\partial^{2} u}{\partial x^{2}}, \quad u(0, x)=\varphi(x), \quad u(t, 0)=\varphi(0), \quad u(t, \pi)=\varphi(\pi) .
$$

$\mathrm{B}$ случае, когда $\operatorname{Re} \sigma^{2}=0$, для того чтобы функция $u(t, x)=P_{D}^{t} \varphi(x)$ являлась решением задачи (32), достаточно, чтобы функция $\varphi_{0}^{\text {odd }}$ принадлежала классу $\mathscr{R}^{(2)}[-\pi, \pi]$.

Получим теперь явное выражение для оператора $P_{D}^{t}$. Сначала рассмотрим случай $\operatorname{Re} \sigma^{2}>0$. 
Лемма 2. Пусть $\operatorname{Re} \sigma^{2}>0$, a оператор $V^{t}: \mathscr{R}[-\pi, \pi] \rightarrow \mathscr{R}[-\pi, \pi]$ переводит каждую функиию $f \in \mathscr{R}[-\pi, \pi]$ вида $f(x)=\sum_{m=-\infty}^{\infty} B_{m} e^{i m x}$ в функиию $V^{t} f$, где

$$
V^{t} f(x)=\sum_{m=-\infty}^{\infty} B_{m} e^{-t \sigma^{2} m^{2} / 2} e^{i m x} .
$$

Тогда справедливы следующие утверждения.

1. Для любого $f \in \mathscr{R}[-\pi, \pi]$

$$
V^{t} f(x)=\int_{-\infty}^{\infty} f(x-y) \psi_{t}(y) d y=f * \psi_{t}(x),
$$

где $\psi_{t}(y)=e^{-y^{2} /\left(2 \sigma^{2} t\right)} /(\sigma \sqrt{2 \pi t})$.

2. Для любого $f \in \mathscr{R}[-\pi, \pi]$

$$
V^{t} f(x)=\int_{-\pi}^{\pi} f(x-y) q_{t}(y) d y=\int_{-\pi}^{\pi} f(y) q_{t}(x-y) d y,
$$

где $q_{t}(y)=\sum_{k=-\infty}^{\infty} \psi_{t}(y-2 \pi k)$.

3. Для каждого $t>0$ оператор $V^{t}$ может быть продолжен до сжимающего оператора в $L_{2}[-\pi, \pi]$, при этом для продолженного оператора формула (34) также справедлива.

Д о к а з а т е л ь с т в о. Для доказательства утверждения 1 достаточно заметить, что функция $u(t, x)=V^{t} f(x)$ является решением задачи Коши

$$
\frac{\partial u}{\partial t}=\frac{\sigma^{2}}{2} \frac{\partial^{2} u}{\partial x^{2}}, \quad u(0, x)=f(x),
$$

и, значит, может быть представлена как свертка функции $f$ с соответствующим фундаментальным решением $\psi_{t}$.

Докажем утверждение 2. Имеем

$$
\begin{aligned}
V^{t} f(x) & =\int_{-\infty}^{\infty} f(x-y) \psi_{t}(y) d y \\
& =\sum_{k=-\infty}^{\infty} \int_{-\pi-2 \pi k}^{\pi-2 \pi k} f(x-y) \psi_{t}(y) d y \\
& =\sum_{k=-\infty}^{\infty} \int_{-\pi}^{\pi} f(x-y) \psi_{t}(y-2 \pi k) d y \\
& =\int_{-\pi}^{\pi} f(x-y) \sum_{k=-\infty}^{\infty} \psi_{t}(y-2 \pi k) d y
\end{aligned}
$$

Утверждение 3 следует из того, что Фурье-образ оператора $V^{t}$ есть оператор умножения на функцию, по модулю не превосходящую единицы. Лемма 2 доказана. 
Рассмотрим теперь случай $\operatorname{Re} \sigma^{2}=0$. Для определенности будем считать, что $\sigma^{2}=i$; случай $\sigma^{2}=-i$ рассматривается аналогично. Функция $\psi_{t}(y)=\left(e^{-i \pi / 4} / \sqrt{2 \pi t}\right) e^{i y^{2} /(2 t)}$ задает линейный функционал (обобщенную функцию) $\psi_{t}$ на $\mathscr{R}^{(2)}[-\pi, \pi]$. Именно, для $g \in \mathscr{R}^{(2)}[-\pi, \pi]$

$$
\left(\psi_{t}, g\right)=\int_{-\infty}^{\infty} g(y) \psi_{t}(y) d y
$$

причем (35) понимается как несобственный (сходящийся, но не абсолютно) интеграл, именно,

$$
\int_{-\infty}^{\infty} g(y) \psi_{t}(y) d y=\lim _{\substack{M \rightarrow \infty \\ N \rightarrow \infty}} \int_{-N}^{M} g(y) \psi_{t}(y) d y
$$

Конечность последнего предела легко проверить двукратным интегрированием по частям.

Лемма 3. Пусть оператор $V^{t}: \mathscr{R}^{(2)}[-\pi, \pi] \rightarrow \mathscr{R}^{(2)}[-\pi, \pi]$ переводит каждую функиию $f \in \mathscr{R}^{(2)}[-\pi, \pi]$ вида $f(x)=\sum_{m=-\infty}^{\infty} B_{m} e^{i m x}$ в функцию $V^{t} f$, где

$$
V^{t} f(x)=\sum_{m=-\infty}^{\infty} B_{m} e^{-i t m^{2} / 2} e^{i m x}
$$

Тогда справедливы следующие утверждения.

1. Для любого $f \in \mathscr{R}^{(2)}[-\pi, \pi]$

$$
V^{t} f(x)=\int_{-\infty}^{\infty} f(x-y) \psi_{t}(y) d y=\int_{-\infty}^{\infty} f(y) \psi_{t}(x-y) d y=f * \psi_{t}(x)
$$

где $\psi_{t}(y)=\left(e^{-i \pi / 4} / \sqrt{2 \pi t}\right) e^{i y^{2} /(2 t)}$.

2. Для любого $f \in \mathscr{R}^{(2)}[-\pi, \pi]$

$$
V^{t} f(x)=\int_{-\pi}^{\pi} f(x-y) q_{t}(y) d y=\int_{-\pi}^{\pi} f(y) q_{t}(x-y) d y,
$$

где $q_{t}(y)=\sum_{k=-\infty}^{\infty} \psi_{t}(y-2 \pi k)$, а сумма ряда понимается в смьсле сходимости обобщенных функиий над классом $\mathscr{R}^{(2)}[-\pi, \pi]$.

3. Для каждого $t>0$ оператор $V^{t}$ может быть продолжен до унитарного оператора в $L_{2}[-\pi, \pi]$.

Д о к а з а т е л ь с т в о. Утверждения 1 и 2 доказываются так же, как в лемме 1 , а утверждение 3 следует из того, что Фурье-образ этого оператора есть оператор умножения на функцию, по модулю равную единице. Лемма 3 доказана. 
Используя (31) и лемму 2 , получаем, что в случае $\operatorname{Re} \sigma^{2}>0$

$$
\begin{aligned}
P_{D}^{t} \varphi(x) & =\varphi(0)+\frac{\varphi(\pi)-\varphi(0)}{\pi} x+\int_{-\pi}^{\pi} \varphi_{0}^{\text {odd }}(y) q_{t}(x-y) d y \\
& =\varphi(0)+\frac{\varphi(\pi)-\varphi(0)}{\pi} x+\int_{0}^{\pi} \varphi_{0}(y)\left[q_{t}(x-y)-q_{t}(x+y)\right] d y \\
& =\varphi(0)\left(1-\frac{x}{\pi}\right)+\varphi(\pi) \frac{x}{\pi}+\int_{0}^{\pi} \varphi_{0}(y) r_{t, x}(y) d y
\end{aligned}
$$

где $r_{t, x}(y)=q_{t}(x-y)-q_{t}(x+y)$.

Далее, в силу (28) имеем

$$
\begin{aligned}
P_{D}^{t} \varphi(x)= & \varphi(0)\left(1-\frac{x}{\pi}-\int_{0}^{\pi} r_{t, x}(y) d y+\frac{1}{\pi} \int_{0}^{\pi} y r_{t, x}(y) d y\right) \\
& +\varphi(\pi)\left(\frac{x}{\pi}-\frac{1}{\pi} \int_{0}^{\pi} y r_{t, x}(y) d y\right)+\int_{0}^{\pi} \varphi(y) r_{t, x}(y) d y .
\end{aligned}
$$

Из (39) следует, что для каждого $t>0$ одномерное распределение (в смысле обобщенных функций) процесса $\xi_{x}^{\sigma}(t)$ в случае, когда продолжение $\varphi \mapsto \widetilde{\varphi}_{D}$ задается формулой $(30)$, для каждого $x \in[0, \pi]$ есть сумма (комплекснозначного) заряда с плотностью $r_{t, x}$ на интервале $[0, \pi]$ и (также комплекснозначного) заряда, сосредоточенного на границе интервала. Кроме того, мы можем определить предельный оператор $P_{D}^{\infty}=\lim _{t \rightarrow+\infty} P_{D}^{t}$, полагая

$$
P_{D}^{\infty} \varphi(x)=\varphi(0)\left(1-\frac{x}{\pi}\right)+\varphi(\pi) \frac{x}{\pi} .
$$

В случае $\sigma^{2}=i$ формула (39) по-прежнему имеет смысл, но $r_{t, x}(y)=$ $q_{t}(x-y)-q_{t}(x+y)$ является только обобщенной функцией, заданной на множестве функций $\varphi$ таких, что нечетное продолжение $\varphi_{0}^{\text {odd }}$ функции $\varphi_{0}$ принадлежит классу $\mathscr{R}^{(2)}[-\pi, \pi]$. Выражения $\int_{0}^{\pi} r_{t, x}(y) d y$ и $\int_{0}^{\pi} y r_{t, x}(y) d y$ понимаются уже только как действие $L_{2}$-продолжения оператора $(36)$ на нечетное продолжение индикаторной функции интервала $[0, \pi]$ и функцию $y$ соответственно. Тем самым, в случае $\operatorname{Re} \sigma^{2}=0$ одномерное распределение процесса $\xi_{x}^{\sigma}(t)$ при всех $t>0$ есть сумма обобщенной функции на интервале $[0, \pi]$ и заряда, сосредоточенного на границе интервала, но в отличие от случая $\operatorname{Re} \sigma^{2}>0$ этот заряд задан только как $L_{2}$-функция начальной точки $x$, т.е. представляет собой по существу класс эквивалентности функций на $[0, \pi]$. Отметим еще, что в случае $\operatorname{Re} \sigma^{2}=0$ не существует предела $\lim _{t \rightarrow+\infty} P_{D}^{t}$.

Рассмотрим теперь, как действует «допредельный» оператор $P_{D, n}^{t}$. Используя (25) и (30), получим

$$
P_{D, n}^{t} \varphi(x)=\mathbf{E}_{n} \widetilde{\varphi}_{D}(x+\sigma \omega[0, t])
$$




$$
\begin{aligned}
= & \mathbf{E}_{n}\left(\varphi(0)+\frac{\varphi(\pi)-\varphi(0)}{\pi}(x+\sigma \omega[0, t])\right) \\
& +\mathbf{E}_{n}\left(\sum_{m=-\infty}^{\infty} B_{m} e^{i m(x+\sigma \omega[0, t])}\right) .
\end{aligned}
$$

Далее, как легко видеть, $\mathbf{E}_{n} \omega[0, t]=0$, а в силу (13) и $(25)$

$$
\begin{aligned}
\mathbf{E}_{n} e^{i m \sigma \omega[0, t]} & =\sum_{k=0}^{\infty} \frac{t^{k}}{k !}\left(g_{n}^{\otimes k}, e^{i m \sigma\left(x_{1}+\cdots+x_{k}\right)}\right) \\
& =\sum_{k=0}^{\infty} \frac{t^{k}}{k !}\left(\frac{n}{\sqrt{2 \pi}} \int_{\mathbf{R}}\left(e^{i m \sigma y / \sqrt{n}}-1\right) e^{-y^{2} / 2} d y\right)^{k} \\
& =e^{n t\left(e^{-m^{2} \sigma^{2} /(2 n)}-1\right)} .
\end{aligned}
$$

Заметим, что из условия $\operatorname{Re} \sigma^{2} \geqslant 0$ следует неравенство

$$
\left|e^{n t\left(e^{-m^{2} \sigma^{2} /(2 n)}-1\right)}\right| \leqslant 1 .
$$

Таким образом, имеем

$$
P_{D, n}^{t} \varphi(x)=\varphi(0)+\frac{\varphi(\pi)-\varphi(0)}{\pi} x+\sum_{m=-\infty}^{\infty} B_{m} e^{n t\left(e^{-m^{2} \sigma^{2} /(2 n)}-1\right)} e^{i m x},
$$

что означает, что оператор $P_{D, n}^{t}$ не меняет линейную часть функции $\varphi$, а его действие на $\varphi_{0}$ сводится к умножению коэффициента Фурье $B_{m}$ на величину $e^{n t\left(e^{-m^{2} \sigma^{2} /(2 n)}-1\right)}$ при всех $m \in \mathbf{Z}$.

Используя (42), легко показать, что для каждой $\varphi$ такой, что $\varphi_{0}^{\text {odd }} \in$ $\mathscr{R}^{(2)}[-\pi, \pi]$, функция $u_{n}(t, x)=P_{D, n}^{t} \varphi(x)$ решает начально-краевую задачу

$$
\frac{\partial u_{n}}{\partial t}=A_{D, n}^{\sigma} u_{n}, \quad u_{n}(0, x)=\varphi(x), \quad u_{n}(t, 0)=\varphi(0), \quad u_{n}(t, \pi)=\varphi(\pi),
$$

где оператор $A_{D, n}^{\sigma}$ действует на функцию $\psi \in \mathscr{H}_{D}$ следующим образом:

$$
\begin{aligned}
A_{D, n}^{\sigma} \psi(x) & =\frac{n}{\sqrt{2 \pi}} \int_{\mathbf{R}}\left(\widetilde{\psi}_{D}\left(x+\frac{\sigma y}{\sqrt{n}}\right)-\widetilde{\psi}_{D}(x)\right) e^{-y^{2} / 2} d y \\
& =\frac{n}{\sqrt{2 \pi}} \int_{\mathbf{R}}\left(\psi_{0}^{\text {odd }}\left(x+\frac{\sigma y}{\sqrt{n}}\right)-\psi_{0}^{\text {odd }}(x)\right) e^{-y^{2} / 2} d y .
\end{aligned}
$$

Отметим, что формулой (44) оператор $A_{D, n}^{\sigma}$ задается только на функциях из $\mathscr{H}_{D}$, поскольку в качестве аргумента функции $\psi$ подставляется комплексное число. Нетрудно показать, однако, что действие оператора $A_{D, n}^{\sigma}$ на функцию $\psi_{0}^{\text {odd }}(x)=\sum_{m=-\infty}^{\infty} B_{m} e^{i m x}$ сводится к умножению коэффициента Фурье $B_{m}$ на величину $n\left(e^{-m^{2} \sigma^{2} /(2 n)}-1\right)$, а из 
условия $\operatorname{Re} \sigma^{2} \geqslant 0$ вытекает, что при всех $m \in \mathbf{Z}$ эта величина по модулю не превосходит $m^{2}$. Отсюда, в свою очередь, вытекает, что оператор $A_{D, n}^{\sigma}$ может быть продолжен на множество функций $\psi$ таких, что $\psi_{0}^{\text {odd }} \in \mathscr{R}^{(2)}[-\pi, \pi]$. Всюду далее под $A_{D, n}^{\sigma}$ мы будем подразумевать именно продолженный оператор.

Аналогичным образом, исходно операторы $P_{D}^{t}$ и $P_{D, n}^{t}$ заданы только на функциях из $\mathscr{H}_{D}$. Однако из лемм 2,3 и соотношений (41), (42) вытекает, что эти операторы можно продолжить на более широкое пространство $\mathscr{R}^{\text {odd }}$ непрерывных функций $\varphi:[0, \pi] \rightarrow \mathbf{C}$ таких, что $\varphi_{0}^{\text {odd }} \in \mathscr{R}[-\pi, \pi]$. Каждая функция $\varphi \in \mathscr{R}^{\text {odd }}$ однозначно представима в виде $\varphi(x)=a+b x+\varphi_{0}(x), \varphi_{0}(0)=\varphi_{0}(\pi)=0$. Определим в этом пространстве норму, полагая

$$
\|\varphi\|_{\mathscr{R} \text { odd }}=|a|+|b| \pi+\sum_{m=-\infty}^{\infty}\left|B_{m}\right| .
$$

Ясно, что $\|\varphi\|_{\infty} \leqslant\|\varphi\|_{\mathscr{R} \text { odd }}$.

В следующей теореме речь идет о сходимости продолженных операторов.

Теорема 3. Пусть $\varphi \in \mathscr{R}^{\text {odd }}$, тогда равномерно по $t \in[0, T]$

$$
\lim _{n \rightarrow \infty}\left\|P_{D, n}^{t} \varphi-P_{D}^{t} \varphi\right\|_{\mathscr{R}}^{\text {odd }}=0 .
$$

Д о к а з а т ель с т в о. Имеем

$$
\left\|P_{D, n}^{t} \varphi-P_{D}^{t} \varphi\right\|_{\mathscr{R} \text { odd }} \leqslant \sum_{m=-\infty}^{\infty}\left|B_{m}\right|\left|e^{n t\left(e^{-m^{2} \sigma^{2} / 2 n}-1\right)}-e^{-t \sigma^{2} m^{2} / 2}\right| .
$$

Последняя сумма стремится к нулю по теореме Лебега о мажорируемой сходимости. Равномерная сходимость по $t \in[0, T]$ легко следует из равномерной (по $t \in[0, T]$ и по $|m| \leqslant M$ для любого фиксированного $M$ ) сходимости функций $e^{n t\left(e^{-m^{2} \sigma^{2} /(2 n)}-1\right)}$ при $n \rightarrow \infty$. Теорема 3 доказана.

4.2. Условие Неймана. Снова будем проводить рассмотрения на интервале $[0, \pi]$. Пусть непрерывно дифференцируемая комплекснозначная функция $\varphi$ определена на интервале $[0, \pi]$. Разложим функцию $\varphi$ в сумму

$$
\varphi(x)=\varphi^{\prime}(0) x+\frac{\varphi^{\prime}(\pi)-\varphi^{\prime}(0)}{2 \pi} x^{2}+\varphi_{0}(x),
$$

при этом $\varphi_{0}^{\prime}(0)=\varphi_{0}^{\prime}(\pi)=0$. Для каждой функции $\varphi_{0}$ через $\varphi_{0}^{\text {even }}$ обозначим ее четное продолжение с $[0, \pi]$ на $[-\pi, \pi]$.

Пусть $\mathscr{H}_{N}$ обозначает множество функций $\varphi:[0, \pi] \rightarrow \mathbf{C}$, у которых в разложении (45) функция $\varphi_{0}^{\text {even }}$ имеет вид

$$
\varphi_{0}^{\mathrm{even}}(x)=\sum_{m=-\infty}^{\infty} B_{m} e^{i m x}
$$


причем $B_{-m}=B_{m}$ для всех $m$ и только конечное число коэффициентов $B_{m}$ отлично от нуля.

Для функции $\varphi \in \mathscr{H}_{N}$ определим ее продолжение $\widetilde{\varphi}_{N}$, полагая

$$
\widetilde{\varphi}_{N}(z)=\varphi^{\prime}(0) z+\frac{\varphi^{\prime}(\pi)-\varphi^{\prime}(0)}{2 \pi} z^{2}+\sum_{m=-\infty}^{\infty} B_{m} e^{i m z} .
$$

Отметим, что при этом продолжении с интервала $[0, \pi]$ на вещественную ось функция $\varphi_{0}$ продолжается сначала четным $($ на $[-\pi, \pi])$, а затем $2 \pi$-периодическим образом.

Определим две полугруппы операторов $P_{N, n}^{t}$ и $P_{N}^{t}(t \geqslant 0)$, полагая для $\varphi \in \mathscr{H}_{N}$

$$
P_{N}^{t} \varphi(x)=\mathbf{L} \widetilde{\varphi}_{N}\left(\xi_{x}^{\sigma}(t)\right), \quad P_{n, N}^{t} \varphi(x)=\mathbf{E}_{n} \widetilde{\varphi}_{N}\left(\xi_{x}^{\sigma}(t)\right) .
$$

Посмотрим, как действует оператор $P_{N}^{t}$. Пусть $\varphi \in \mathscr{H}_{N}$. Используя лемму 1 , получим

$$
\begin{aligned}
P_{N}^{t} \varphi(x)= & \mathbf{L}^{0, t} \widetilde{\varphi}_{N}(x+\sigma \omega[0, t]) \\
= & \varphi^{\prime}(0) x+\frac{\varphi^{\prime}(\pi)-\varphi^{\prime}(0)}{2 \pi}\left(x^{2}+\sigma^{2} t\right) \\
& +\sum_{m=-\infty}^{\infty} B_{m} e^{-t \sigma^{2} m^{2} / 2} e^{i m x} .
\end{aligned}
$$

Исходно оператор $P_{N}^{t}$ задан только на функциях из $\mathscr{H}_{N}$, но с помощью (48) он продолжается на множество функций $\varphi$ таких, что $\varphi_{0}^{\text {even }} \in \mathscr{R}^{(1)}[-\pi, \pi]$.

При этом, если $\operatorname{Re} \sigma^{2}>0$, то для каждой такой функции $\varphi$ функция $u(t, x)=P_{N}^{t} \varphi(x)$ (здесь речь идет о продолженном операторе) является решением начально-краевой задачи

$$
\frac{\partial u}{\partial t}=\frac{\sigma^{2}}{2} \frac{\partial^{2} u}{\partial x^{2}}, \quad u(0, x)=\varphi(x), \quad \frac{\partial u}{\partial x}(t, 0)=\varphi^{\prime}(0), \quad \frac{\partial u}{\partial x}(t, \pi)=\varphi^{\prime}(\pi) .
$$

В случае $\operatorname{Re} \sigma^{2}=0$ функция $u(t, x)=P_{N}^{t} \varphi(x)$ есть решение задачи (49), если $\varphi_{0}^{\text {even }} \in \mathscr{R}^{(2)}[-\pi, \pi]$.

Используя леммы 2 и 3 , получаем

$$
\begin{aligned}
P_{N}^{t} \varphi(x) & =\varphi^{\prime}(0) x+\frac{\varphi^{\prime}(\pi)-\varphi^{\prime}(0)}{2 \pi}\left(x^{2}+\sigma^{2} t\right)+\int_{-\pi}^{\pi} \varphi_{0}^{\text {even }}(y) q_{t}(x-y) d y \\
& =\varphi^{\prime}(0)\left(x-\frac{x^{2}+\sigma^{2} t}{2 \pi}\right)+\varphi^{\prime}(\pi) \frac{x^{2}+\sigma^{2} t}{2 \pi}+\int_{0}^{\pi} \varphi_{0}(y) \widetilde{r}_{t, x}(y) d y,
\end{aligned}
$$

где $\widetilde{r}_{t, x}(y)=q_{t}(x-y)+q_{t}(x+y)$. 
Далее, в силу (45) имеем

$$
\begin{aligned}
P_{N}^{t} \varphi(x)= & \varphi^{\prime}(0)\left(x-\frac{x^{2}+\sigma^{2} t}{2 \pi}-\int_{0}^{\pi} y \widetilde{r}_{t, x}(y) d y+\frac{1}{2 \pi} \int_{0}^{\pi} y^{2} \widetilde{r}_{t, x}(y) d y\right) \\
& +\varphi^{\prime}(\pi)\left(\frac{x^{2}+\sigma^{2} t}{2 \pi}-\frac{1}{2 \pi} \int_{0}^{\pi} y^{2} \widetilde{r}_{t, x}(y) d y\right) \\
& +\int_{0}^{\pi} \varphi(y) \widetilde{r}_{t, x}(y) d y
\end{aligned}
$$

В случае $\operatorname{Re} \sigma^{2}>0$ из формулы (50) следует, что для каждого $t$, $t>0$, одномерное распределение (в смысле обобщенных функций) процесса $\xi_{x}^{\sigma}(t)$ в предположении, что продолжение $\varphi \mapsto \widetilde{\varphi}_{N}$ задается формулой $(47)$, для каждого $x \in[0, \pi]$ есть сумма заряда с плотностью $\widetilde{r}_{t, x}$ на интервале $[0, \pi]$ и обобщенной функции с носителем на границе интервала. Предельный оператор $P_{N}^{\infty}=\lim _{t \rightarrow+\infty} P_{N}^{t}$ определен только на множестве функций, удовлетворяющих условию $\varphi^{\prime}(0)=\varphi^{\prime}(\pi)$, и задается формулой

$$
P_{N}^{\infty} \varphi(x)=\varphi^{\prime}(0)\left(x-\frac{\pi}{2}\right)+\frac{1}{\pi} \int_{0}^{\pi} \varphi(y) d y .
$$

В случае $\operatorname{Re} \sigma^{2}=0$ в формуле (50) $\widetilde{r}_{t, x}=q_{t}(x-y)+q_{t}(x+y)$ является только обобщенной функцией, заданной на множестве функций $\varphi$ таких, что их четное продолжение $\varphi^{\text {even }}$ принадлежит классу $\mathscr{R}^{(2)}[-\pi, \pi]$, а выражения $\int_{0}^{\pi} y \widetilde{r}_{t, x}(y) d y$ и $\int_{0}^{\pi} y^{2} \widetilde{r}_{t, x}(y) d y$ понимаются уже только как действие $L_{2}$-продолжения оператора $(36)$ на четное продолжение функции $y$ и четное продолжение функции $y^{2}$ соответственно. При этом не существует предела $\lim _{t \rightarrow+\infty} P_{N}^{t}$.

Рассмотрим теперь, как действует «допредельный» оператор $P_{N, n}^{t}$. Используя (25) и (47), получим

$$
\begin{aligned}
P_{N, n}^{t} \varphi(x)= & \mathbf{E}_{n} \widetilde{\varphi}_{N}(x+\sigma \omega[0, t]) \\
= & \mathbf{E}_{n}\left(\varphi^{\prime}(0)(x+\sigma \omega[0, t])+\frac{\varphi^{\prime}(\pi)-\varphi^{\prime}(0)}{2 \pi}(x+\sigma \omega[0, t])^{2}\right) \\
& +\mathbf{E}_{n}\left(\sum_{m=-\infty}^{\infty} B_{m} e^{i m(x+\sigma \omega[0, t])}\right) \\
= & \varphi^{\prime}(0) x+\frac{\varphi^{\prime}(\pi)-\varphi^{\prime}(0)}{2 \pi}\left(x^{2}+\sigma^{2} t\right) \\
& +\sum_{m=-\infty}^{\infty} B_{m} e^{n t\left(e^{-m^{2} \sigma^{2} /(2 n)}-1\right)} e^{i m x} .
\end{aligned}
$$

Используя (51), легко показать, что для каждой $\varphi$ такой, что $\varphi_{0}^{\text {even }} \in$ $\mathscr{R}^{(2)}[-\pi, \pi]$, функция $u_{n}(t, x)=P_{n, N}^{t} \varphi(x)$ решает начально-краевую за- 
дачу

$$
\begin{gathered}
\frac{\partial u_{n}}{\partial t}=A_{N, n}^{\sigma} u_{n}, \quad u_{n}(0, x)=\varphi(x), \\
\frac{\partial u_{n}}{\partial x}(t, 0)=\varphi^{\prime}(0), \quad \frac{\partial u_{n}}{\partial x}(t, \pi)=\varphi^{\prime}(\pi),
\end{gathered}
$$

где оператор $A_{N, n}^{\sigma}$ действует на функцию $\psi \in \mathscr{H}_{N}$ следующим образом:

$$
\begin{aligned}
& A_{N, n}^{\sigma} \psi(x)=\frac{n}{\sqrt{2 \pi}} \int_{\mathbf{R}}\left(\widetilde{\psi}_{N}\left(x+\frac{\sigma y}{\sqrt{n}}\right)-\widetilde{\psi}_{N}(x)\right) e^{-y^{2} / 2} d y \\
& \quad=\frac{\varphi^{\prime}(\pi)-\varphi^{\prime}(0)}{2 \pi} \sigma^{2}+\frac{n}{\sqrt{2 \pi}} \int_{\mathbf{R}}\left(\psi_{0}^{\text {even }}\left(x+\frac{\sigma y}{\sqrt{n}}\right)-\psi_{0}^{\text {even }}(x)\right) e^{-y^{2} / 2} d y .
\end{aligned}
$$

Оператор $A_{N, n}^{\sigma}$ исходно задан только на функциях из $\mathscr{H}_{N}$, но так же, как для оператора (44), можно показать, что $A_{N, n}^{\sigma}$ может быть продолжен на множество функций $\psi$ таких, что $\psi_{0}^{\text {even }} \in \mathscr{R}^{(2)}[-\pi, \pi]$.

Далее, исходно операторы $P_{N}^{t}$ и $P_{n, N}^{t}$ также заданы только на функциях из $\mathscr{H}_{N}$. Однако эти операторы могут быть продолжены на более широкое пространство $\mathscr{R}^{\text {even }}$ непрерывных функций $\varphi:[0, \pi] \rightarrow \mathbf{C}$ таких, что $\varphi_{0}^{\text {even }} \in \mathscr{R}^{(1)}[-\pi, \pi]$. Каждая функция $\varphi \in \mathscr{R}^{\text {even }}$ однозначно представима в виде $\varphi(x)=a x+b x^{2}+\varphi_{0}(x), \varphi_{0}^{\prime}(0)=\varphi_{0}^{\prime}(\pi)=0$. Определим в этом пространстве норму, полагая

$$
\|\varphi\|_{\mathscr{R}^{\mathrm{even}}}=(\pi+1)|a|+\left(\pi^{2}+2 \pi\right)|b|+\sum_{m=-\infty}^{\infty}(1+|m|)\left|B_{m}\right| .
$$

Ясно, что $\|\varphi\|_{\infty}+\left\|\varphi^{\prime}\right\|_{\infty} \leqslant\|\varphi\|_{\mathscr{R} \text { even }}$

В следующей теореме речь идет о сходимости продолженных операторов.

Теорема 4. Пусть $\varphi \in \mathscr{R}^{\mathrm{even}}$, тогда равномерно по $t \in[0, T]$

$$
\lim _{n \rightarrow \infty}\left\|P_{N, n}^{t} \varphi-P_{N}^{t} \varphi\right\|_{\mathscr{R}^{\text {even }}}=0 .
$$

Д о к а з а т е ль с т в о. Имеем

$$
\left\|P_{N, n}^{t} \varphi-P_{N}^{t} \varphi\right\|_{\mathscr{R}}{ }^{\text {ven }} \leqslant \sum_{m=-\infty}^{\infty}(1+|m|)\left|B_{m}\right|\left|e^{n t\left(e^{-m^{2} \sigma^{2} /(2 n)}-1\right)}-e^{-t \sigma^{2} m^{2} / 2}\right| .
$$

Последняя сумма стремится к нулю по теореме Лебега о мажорируемой сходимости. Теорема 4 доказана.

5. Представление решения уравнения с ограниченным потенциалом. Как было показано выше, для каждой $\varphi$ такой, что $\varphi_{0}^{\text {odd }} \in$ $\mathscr{R}^{(2)}[-\pi, \pi]$, функция $u(t, x)=P_{D}^{t} \varphi(x)=\mathbf{L} \widetilde{\varphi}_{D}\left(\xi_{x}^{\sigma}(t)\right)$ является решением начально-краевой задачи (32). Теперь мы хотим получить аналогичное представление, но уже для уравнения, возмущенного ограниченным 
потенциалом. Именно, рассмотрим начально-краевую задачу (мы ограничимся только случаем условия Дирихле на границе)

$$
\frac{\partial u}{\partial t}=\frac{\sigma^{2}}{2} \frac{\partial^{2} u}{\partial x^{2}}+f(x) u, \quad u(0, x)=\varphi(x), \quad u(t, 0)=u(t, \pi)=0
$$

и получим для решения этой задачи вероятностное представление, аналогичное представлению решения невозмущенного уравнения.

Пусть $\widetilde{f}^{\text {even }}$ обозначает четное $(\mathrm{c}[0, \pi]$ на $[-\pi, \pi])$ продолжение функции $f$. Предположим сначала, что $\widetilde{f}^{\text {even }}$ - тригонометрический полином вида (46). Определим два семейства операторов $F_{D, n}^{t}$ и $F_{D}^{t}(t \geqslant 0)$, полагая для $\varphi \in \mathscr{H}_{D}$

$$
\begin{aligned}
F_{D}^{t} \varphi(x) & =\mathbf{L}\left[\widetilde{\varphi}_{D}\left(\xi_{x}^{\sigma}(t)\right) \exp \left(\int_{0}^{t} \tilde{f}^{\text {even }}\left(\xi_{x}^{\sigma}(v)\right) d v\right)\right] \\
& =\mathbf{L}^{0, t}\left[\widetilde{\varphi}_{D}\left(\xi_{x}^{\sigma}(t)\right) \exp \left(\int_{0}^{t} \tilde{f}^{\text {even }}\left(\xi_{x}^{\sigma}(v)\right) d v\right)\right], \\
F_{D, n}^{t} \varphi(x) & =\mathbf{E}_{n}\left[\widetilde{\varphi}_{D}\left(\xi_{x}^{\sigma}(t)\right) \exp \left(\int_{0}^{t} \tilde{f}^{\text {even }}\left(\xi_{x}^{\sigma}(v)\right) d v\right)\right] .
\end{aligned}
$$

Покажем, что введенные семейства образуют полугруппу. В силу соотношения (22) имеем

$$
\begin{aligned}
F_{D}^{t+s} \varphi(x)= & \mathbf{L}\left[\widetilde{\varphi}_{D}\left(\xi_{x}^{\sigma}(t+s)\right) \exp \left(\int_{0}^{t+s} \tilde{f}^{\text {even }}\left(\xi_{x}^{\sigma}(v)\right) d v\right)\right] \\
= & \mathbf{L}^{0, t}\left[\operatorname { e x p } ( \int _ { 0 } ^ { t } \tilde { f } ^ { \text { even } } ( \xi _ { x } ^ { \sigma } ( v ) ) d v ) \mathbf { L } ^ { t , t + s } \left[\widetilde{\varphi}_{D}\left(\xi_{x}^{\sigma}(t+s)\right)\right.\right. \\
& \left.\left.\quad \times \exp \left(\int_{t}^{t+s} \widetilde{f}^{\text {even }}\left(\xi_{x}^{\sigma}(v)\right) d v\right)\right]\right] \\
= & \mathbf{L}^{0, t}\left[\exp \left(\int_{0}^{t} \tilde{f}^{\text {even }}\left(\xi_{x}^{\sigma}(v)\right) d v\right)\left(F_{D}^{s} \varphi\right)\left(\xi_{x}^{\sigma}(t)\right)\right] \\
= & F_{D}^{t} F_{D}^{s} \varphi(x) .
\end{aligned}
$$

Аналогичным образом доказывается справедливость полугруппового свойства для семейства $F_{D, n}^{t}$.

Следующим шагом найдем генераторы введенных полугрупп.

Лемма 4. Пусть $\varphi \in \mathscr{H}_{D}$, a $\tilde{f}^{\text {even }}-$ тригонометрический полином вида (46). Тогда для любого $x \in(0, \pi)$ при $t \rightarrow 0+$

$$
\begin{aligned}
F_{D}^{t} \varphi(x) & =\varphi(x)+\frac{\sigma^{2}}{2} \varphi^{(2)}(x) t+\varphi(x) f(x) t+o(t), \\
F_{D, n}^{t} \varphi(x) & =\varphi(x)+A_{D, n}^{\sigma} \varphi(x) t+\varphi(x) f(x) t+o(t),
\end{aligned}
$$

где оператор $A_{D, n}^{\sigma}$ определен формулой (44). 
Д о к а з а т е л ь с т в о. В силу (16) имеем

$$
\begin{aligned}
F_{D}^{t} \varphi(x)= & \mathbf{L}\left[\widetilde{\varphi}_{D}\left(\xi_{x}^{\sigma}(t)\right) \exp \left\{\int_{0}^{t} \widetilde{f}^{\text {even }}\left(\xi_{x}^{\sigma}(v)\right) d v\right\}\right] \\
= & \sum_{k=0}^{\infty} \int_{0 \leqslant t_{1}<\cdots<t_{k}<t} d t_{1} \cdots d t_{k}\left(\left(\frac{\delta^{(2)}}{2}\right)^{\otimes k}, \widetilde{\varphi}_{D}\left(x+\sigma\left(x_{1}+\cdots+x_{k}\right)\right)\right. \\
& \left.\times \exp \left\{\sum_{j=1}^{k+1} \widetilde{f}^{\text {even }}\left(x+\sigma\left(x_{1}+\cdots+x_{j-1}\right)\right)\left(t_{j}-t_{j-1}\right)\right\}\right)
\end{aligned}
$$

(в последней сумме по $j$ полагаем $t_{0}=0, t_{k+1}=t$ ).

Ясно, что в сумме по $k$ все слагаемые, кроме $k=0$ и $k=1$, дают вклад $o(t)$. Кроме того, в окрестности точки $x \in(0, \pi)$ мы имеем $\widetilde{\varphi}_{D}(x)=$ $\varphi(x)$ и $\widetilde{f}^{\text {even }}(x)=f(x)$. Таким образом,

$$
\begin{aligned}
F_{D}^{t} \varphi(x) & =\varphi(x) e^{f(x) t}+\int_{0}^{t}\left(\frac{\delta^{(2)}}{2}, \varphi\left(x+\sigma x_{1}\right) e^{f(x) t_{1}+f\left(x+\sigma x_{1}\right)\left(t-t_{1}\right)}\right) d t_{1}+o(t) \\
& =\varphi(x)+\frac{\sigma^{2}}{2} \varphi^{(2)}(x) t+\varphi(x) f(x) t+o(t) .
\end{aligned}
$$

Итак, (54) доказано. Соотношение (55) доказывается аналогично, только вместо формулы (16) надо использовать формулу (15). Лемма 4 доказана.

Из леммы 4 вытекает, что если $\widetilde{f}^{\text {even }}$ - тригонометрический полином вида (46), то для любой функции $\varphi \in \mathscr{H}_{D}$, удовлетворяющей условию $\varphi(0)=\varphi(\pi)=0$, функция $u(t, x)=F_{D}^{t} \varphi(x)$ является решением начально-краевой задачи (53), а функция $u_{n}(t, x)=F_{D, n}^{t} \varphi(x)-$ решением начально-краевой задачи

$$
\frac{\partial u}{\partial t}=A_{D, n}^{\sigma} u+f(x) u, \quad u(0, x)=\varphi(x), \quad u(t, 0)=u(t, \pi)=0
$$

(оператор $A_{D, n}^{\sigma}$ определен формулой (44)).

Покажем теперь, что введенные полугруппы операторов могут быть продолжены на более широкий класс функций $\varphi$ и потенциалов $f$.

Лемма 5. Пусть $g, \psi$ - тригонометрические полиномы вида $\sum_{m} B_{m} e^{i m x}$. Тогда для любого $r \in \mathbf{N}$ справедливы формульь

$$
\begin{aligned}
\mathbf{L} & {\left[\psi\left(\xi_{x}^{\sigma}(t)\right)\left(\int_{0}^{t} g\left(\xi_{x}^{\sigma}(v)\right) d v\right)^{r}\right] } \\
& =r ! \int_{0 \leqslant v_{1} \leqslant \cdots \leqslant v_{r} \leqslant t} \mathbf{L}\left[g\left(\xi_{x}^{\sigma}\left(v_{1}\right)\right) \cdots g\left(\xi_{x}^{\sigma}\left(v_{r}\right)\right) \psi\left(\xi_{x}^{\sigma}(t)\right)\right] d v_{1} \cdots d v_{r}
\end{aligned}
$$

$u$

$$
\begin{aligned}
& \mathbf{E}_{n}\left[\psi\left(\xi_{x}^{\sigma}(t)\right)\left(\int_{0}^{t} g\left(\xi_{x}^{\sigma}(v)\right) d v\right)^{r}\right] \\
& \quad=r ! \int_{0 \leqslant v_{1} \leqslant \cdots \leqslant v_{r} \leqslant t} \mathbf{E}_{n}\left[g\left(\xi_{x}^{\sigma}\left(v_{1}\right)\right) \cdots g\left(\xi_{x}^{\sigma}\left(v_{r}\right)\right) \psi\left(\xi_{x}^{\sigma}(t)\right)\right] d v_{1} \cdots d v_{r} .
\end{aligned}
$$


Д о к а з а т е л ь с т в о. Докажем сначала (58). Имеем

$$
\begin{aligned}
\mathbf{E}_{n} & {\left[\psi\left(\xi_{x}^{\sigma}(t)\right)\left(\int_{0}^{t} g\left(\xi_{x}^{\sigma}(v)\right) d v\right)^{r}\right] } \\
= & r ! \mathbf{E}_{n} \int_{0 \leqslant v_{1} \leqslant \cdots \leqslant v_{r} \leqslant t} g\left(\xi_{x}^{\sigma}(v)\right) \cdots g\left(\xi_{x}^{\sigma}\left(v_{r}\right) \psi\left(\xi_{x}^{\sigma}(t)\right)\right) d v_{1} \cdots d v_{r} \\
& =r ! \int_{0 \leqslant v_{1} \leqslant \cdots \leqslant v_{r} \leqslant t} \mathbf{E}_{n}\left[g\left(\xi_{x}^{\sigma}\left(v_{1}\right)\right) \cdots g\left(\xi_{x}^{\sigma}\left(v_{r}\right)\right) \psi\left(\xi_{x}^{\sigma}(t)\right)\right] d v_{1} \cdots d v_{r} .
\end{aligned}
$$

Таким образом, (58) доказано. Соотношение (57) получается из (58) предельным переходом при $n \rightarrow \infty$. Лемма 5 доказана.

Далее, для функции $\varphi \in \mathscr{H}_{D}$ такой, что $\varphi(0)=\varphi(\pi)=0$, положим $u(t, x)=F_{D}^{t} \varphi(x)$. Используя (58), получаем

$$
\begin{aligned}
& u(t, x)=F_{D}^{t} \varphi(x)=\mathbf{L}\left[\widetilde{\varphi}_{D}\left(\xi_{x}^{\sigma}(t)\right) \exp \left\{\int_{0}^{t} \tilde{f}^{\text {even }}\left(\xi_{x}^{\sigma}(v)\right) d v\right\}\right] \\
& =\mathbf{L}\left[\widetilde{\varphi}_{D}\left(\xi_{x}^{\sigma}(t)\right) \sum_{r=0}^{\infty} \frac{1}{r !}\left(\int_{0}^{t} \tilde{f}^{\text {even }}\left(\xi_{x}^{\sigma}(v)\right) d v\right)^{r}\right] \\
& =\sum_{r=0}^{\infty} \frac{1}{r !} \mathbf{L}\left[\widetilde{\varphi}_{D}\left(\xi_{x}^{\sigma}(t)\right)\left(\int_{0}^{t} \widetilde{f}^{\text {even }}\left(\xi_{x}^{\sigma}(v)\right) d v\right)^{r}\right] \\
& =\sum_{r=0}^{\infty} \int_{0 \leqslant v_{1} \leqslant \cdots \leqslant v_{r} \leqslant t} d v_{1} \cdots d v_{r} \mathbf{L}\left[\widetilde{f}^{\text {even }}\left(\xi_{x}^{\sigma}\left(v_{1}\right)\right) \cdots \widetilde{f}^{\text {even }}\left(\xi_{x}^{\sigma}\left(v_{r}\right)\right) \widetilde{\varphi}_{D}\left(\xi_{x}^{\sigma}(t)\right)\right] \\
& =\sum_{r=0}^{\infty} \int_{0 \leqslant v_{1} \leqslant \cdots \leqslant v_{r} \leqslant t} d v_{1} \cdots d v_{r} P_{D}^{v_{1}}\left(f P_{D}^{v_{2}-v_{1}}\left(f P_{D}^{v_{3}-v_{2}}\left(\ldots f P_{D}^{t-v_{r}} \varphi \ldots\right)\right)(x) .\right.
\end{aligned}
$$

Аналогичным образом,

$$
\begin{aligned}
& u_{n}(t, x)=F_{D, n}^{t} \varphi(x)=\mathbf{E}_{n}\left[\widetilde{\varphi}_{D}\left(\xi_{x}^{\sigma}(t)\right) \exp \left\{\int_{0}^{t} \widetilde{f}^{\text {even }}\left(\xi_{x}^{\sigma}(v) d v\right\}\right]\right. \\
& =\sum_{r=0}^{\infty} \int_{0 \leqslant v_{1} \leqslant \cdots \leqslant v_{r} \leqslant t} d v_{1} \cdots d v_{r} P_{D, n}^{v_{1}}\left(f P_{D, n}^{v_{2}-v_{1}}\left(f P_{D, n}^{v_{3}-v_{2}}\left(\ldots f P_{D, n}^{t-v_{r}} \varphi \ldots\right)\right)\right)(x)
\end{aligned}
$$

Пользуясь формулами (59), (60), мы можем продолжить операторы $F_{D}^{t}, F_{D, n}^{t}$ на более широкий класс функций $\varphi$ и потенциалов $f$.

Лемма 6. Пусть $\tilde{f}^{\text {even }}, \widetilde{\varphi}_{D} \in \mathscr{R}[-\pi, \pi] u \varphi(0)=\varphi(\pi)=0$. Тогда ряды (59), (60) сходятся в $\mathscr{R}[-\pi, \pi]$ равномерно по $t \in[0, T]$.

Д ок а з а т е ль с т в о. Воспользуемся следующими простыми оценками. Пусть $\psi_{1}, \psi_{2} \in \mathscr{R}[-\pi, \pi]$ и $\psi_{1}(0)=\psi_{2}(\pi)=0$. Тогда для любого $t \in[0, T]$

$$
\left\|P_{D}^{t}\left(\psi_{1} \psi_{2}\right)\right\|_{\mathscr{R}} \leqslant\left\|\psi_{1} \psi_{2}\right\|_{\mathscr{R}} \leqslant\left\|\psi_{1}\right\|_{\mathscr{R}}\left\|\psi_{2}\right\|_{\mathscr{R}}
$$


и, аналогично,

$$
\left\|P_{D, n}^{t}\left(\psi_{1} \psi_{2}\right)\right\|_{\mathscr{R}} \leqslant\left\|\psi_{1} \psi_{2}\right\|_{\mathscr{R}} \leqslant\left\|\psi_{1}\right\|_{\mathscr{R}}\left\|\psi_{2}\right\|_{\mathscr{R}} .
$$

Применяя последовательно эти оценки, получаем, что норма слагаемого с номером $r$ в (59) и (60) для любых $v_{1}<\cdots<v_{r}$ оценивается сверху величиной $\left\|\widetilde{\varphi}_{D}\right\|_{\mathscr{R}}\|f\|_{\mathscr{R}}^{r}$. Интегрирование по $v_{1}, \ldots, v_{r}$ даст нам множитель $t^{r} / r$, что гарантирует сходимость рядов (59), (60). Лемма 6 доказана.

Tеорема 5. Пусть $\tilde{f}^{\text {even }} \in \mathscr{R}[-\pi, \pi], \widetilde{\varphi}_{D} \in \mathscr{R}^{(2)}[-\pi, \pi], \varphi(0)=$ $\varphi(\pi)=0$. Тогда функиия $u(t, x)=F_{D}^{t} \varphi(x)$ является решением задачи (53), а функиия $u_{n}(t, x)=F_{D, n}^{t} \varphi(x)$ - решением задачи (56).

Д о к а з а т е л ь с в в. Заметим, что в данном случае речь идет уже о продолженных формулами (59) и (60) операторах. Чтобы сосчитать генераторы соответствующих полугрупп, заметим, что в (59), (60) можно ограничиться только слагаемыми с номерами $r=0$ и $r=1$. При $r=0$ имеем

$$
\begin{aligned}
P_{D}^{t} \varphi(x) & =\varphi(x)+\frac{\sigma^{2}}{2} \varphi^{(2)}(x) t+o(t), \\
P_{D, n}^{t} \varphi(x) & =\varphi(x)+A_{D, n}^{\sigma} \varphi(x)+o(t),
\end{aligned}
$$

а при $r=1$ имеем

$$
\begin{aligned}
\int_{0}^{t} d v_{1} P_{D}^{t}\left(f P_{D}^{t-v_{1}} \varphi\right)(x) & =f(x) \varphi(x) t+o(t), \\
\int_{0}^{t} d v_{1} P_{D, n}^{t}\left(f P_{D, n}^{t-v_{1}} \varphi\right)(x) & =f(x) \varphi(x) t+o(t) .
\end{aligned}
$$

Теорема 5 доказана.

Займемся теперь вопросами сходимости $u_{n} \rightarrow u$.

Теорема 6. Пусть $\tilde{f}^{\text {even }}, \widetilde{\varphi}_{D} \in \mathscr{R}[-\pi, \pi] u \varphi(0)=\varphi(\pi)=0$. Положим $u_{n}(t, x)=F_{D, n}^{t} \varphi(x), u(t, x)=F_{D}^{t} \varphi(x)$. Тогда равномерно по $t \in[0, T]$

$$
\left\|u_{n}(t, \cdot)-u(t, \cdot)\right\|_{\mathscr{R}} \rightarrow 0, \quad n \rightarrow \infty .
$$

Д ок а з а тель с т в о. Заметим прежде всего, что в силу леммы 5 нам достаточно доказать сходимость в $\mathscr{R}[-\pi, \pi]$ отдельных слагаемых суммы (60) к соответствующим слагаемым суммы (59). Последнее утверждение легко доказывается последовательным применением следующей леммы.

Лемма 7. Пусть $\left\{\psi_{n}\right\}$ - последовательность функиий на $[0, \pi]$, причем при всех $n$ вьполняяются условия $\psi_{n}(0)=\psi_{n}(\pi)=0 u\left(\widetilde{\psi}_{n}\right)_{D} \in$

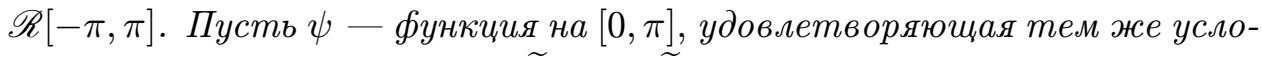
виям. Предположим, ито $\left\|\left(\widetilde{\psi}_{n}\right)_{D}-\widetilde{\psi}_{D}\right\| \mathscr{R} \rightarrow 0, n \rightarrow \infty$. Тогда равномерно по $t \in[0, T]$

$$
\left\|P_{D, n}^{t} \psi_{n}-P_{D}^{t} \psi\right\|_{\mathscr{R}} \rightarrow 0, \quad n \rightarrow \infty .
$$


Д о к а з а те ль с т в о. Имеем

$$
\begin{aligned}
\left\|P_{D, n}^{t} \psi_{n}-P_{D}^{t} \psi\right\|_{\mathscr{R}} & \leqslant\left\|P_{D, n}^{t}\left(\psi_{n}-\psi\right)\right\|_{\mathscr{R}}+\left\|P_{D, n}^{t} \psi-P_{D}^{t} \psi\right\|_{\mathscr{R}} \\
& \leqslant\left\|\psi_{n}-\psi\right\|_{\mathscr{R}}+\left\|P_{D, n}^{t} \psi-P^{t} \psi\right\|_{\mathscr{R}} .
\end{aligned}
$$

Заметим, что второе слагаемое стремится к нулю по теореме 3 . Лемма 7 , а с ней и теорема 6 доказаны.

\section{СПИСОК ЛИТЕРАТУРЫ}

1. Вентчель А. Д. Курс теории случайных процессов. М.: Наука, 1975, 319 с.

2. Скороход A.B. Стохастические уравнения для процессов диффузии с границами. - Теория вероятн. и ее примен., 1961, т. 6, в. 3, с. 287-298.

3. Chung K. L., Zhao Z.X From Brownian Motion to Schrödinger's Equation. BerlinHeidelberg: Springer-Verlag, 1995, 287 p.

4. Freidlin M. Functional Integration and Partial Differential Equations. Princeton: Princeton Univ. Press, 1985, 545 p.

5. Далецкий Ю. Л., Фомин С. В. Меры и дифференциальные уравнения в бесконечномерных пространствах. М: Наука, 1983, 383 с.

6. Глимм Дж., Джаффе А. Математические методы квантовой физики: Подход с использованием функциональных интегралов. М.: Мир, 1984, 445 с.

7. Рид М., Саймон Б. Методы современной математической физики, т. 2. М.: Мир, 1978, 396 c.

8. Doss $H$. Sur une résolution stochastique de l'équation de Schrödinger à coefficients analytiques. - Comm. Math. Phys., 1980, v. 73, № 4, p. 247-264.

9. Albeverio S., Høegh-Krohn R. J., Mazzucchi S. Mathematical theory of Feynman path integrals. - Lecture Notes in Math., 2008, v. 523, p. 1-176.

10. Thaler $H$. Solution of Schrödinger equations on compact Lie groups via probabilistic methods. - Potential Anal., 2003, v. 18, № 2, p. 119-140.

11. Маслов В. П. Комплексные марковские цепи и континуальный интеграл Фейнмана. М.: Наука, 1976, 191 с.

12. Маслов В.П., Чеботарев А. М. Определение континуального интеграла Фейнмана в p-представлении. - Докл. АН СССР, 1976, т. 229, № 1, с. 37-38.

13. Чеботарев A.M. О представлении решения уравнения Шредингера в виде математического ожидания функционалов скачкообразного процесса. - Матем. заметки, 1978, т. 24, № 5, с. 699-706.

14. Albeverio S., Smorodina N., Faddeev M. The probabilistic representation of the exponent of a class of pseudo-differential operators. - J. Global Stoch. Anal., 2011, v. 1, № 2, p. 123-148.

15. Ибрагимов И. А., Смородина Н. В., Фаддеев М. М. Вероятностная аппроксимация решений задачи Коши для некоторых эволюционных уравнений. - Зап. научн. сем. ПОМИ, 2011, т. 396, с. 111-143.

16. Гельфанд И. М., Шилов Г.Е. Обобщенные функции и действия над ними. М.: Физматгиз, 1958, 439 с.

17. Гихман И. И., Скороход А. В. Теория случайных процессов, т. 1. М.: Наука, 1971, $664 \mathrm{c}$. 